\title{
Elastic form factors of nucleon excitations in lattice QCD
}

\author{
Finn M. Stokes $\odot,{ }^{1,2, *}$ Waseem Kamleh ${ }^{1},{ }^{1}$ and Derek B. Leinweber ${ }^{1}$ \\ ${ }^{1}$ Special Research Centre for the Subatomic Structure of Matter, Department of Physics, \\ University of Adelaide, South Australia 5005, Australia \\ ${ }^{2}$ Jülich Supercomputing Centre, Institute for Advanced Simulation, \\ Forschungszentrum Jülich, Jülich D-52425, Germany
}

(Received 12 July 2019; revised 14 May 2020; accepted 25 June 2020; published 15 July 2020)

\begin{abstract}
First principles calculations of the form factors of baryon excitations are now becoming accessible through advances in lattice-QCD techniques. In this paper, we explore the utility of the parity-expanded variational analysis (PEVA) technique in calculating the Sachs electromagnetic form factors for excitations of the proton and neutron. We study the two lowest-lying odd-parity excitations and demonstrate that at heavier quark masses, these states are dominated by behavior consistent with constituent quark models for the $N^{*}(1535)$ and $N^{*}(1650)$, respectively. We also study the lowest-lying localized even-parity excitation and find that its form factors are consistent with a radial excitation of the ground-state nucleon. A comparison of the results from the PEVA technique with those from a conventional variational analysis exposes the necessity of the PEVA approach in baryon excited-state studies.
\end{abstract}

DOI: $10.1103 /$ PhysRevD.102.014507

\section{INTRODUCTION}

Investigating the structure of hadronic excited states is recognized as an important frontier in the field of nonperturbative QCD. At present, very little is known about how QCD composes the structure of these excitations. With regard to the excitations of the nucleon investigated herein, most of our intuition is based on models of QCD as opposed to QCD itself. The intriguing question is, how does the quantum field theory of QCD construct these states and how does this composition compare with the expectations of current models? Can something as simple as a constituent quark model capture the essence of these states? What role do meson-baryon dressings play in describing these states? Our aim is to address these most fundamental questions by examining the electromagnetic structure of nucleon excited states as observed in lattice QCD. The results are fascinating, validating quark-model predictions in some cases and demanding a more important role for meson-baryon interactions in others.

A longer-term goal is to confront experiment. While experimental measurements of resonance transition amplitudes have been made, it is much harder to measure elastic form factors in the resonance regime. This is because elastic

\footnotetext{
*Corresponding author. f.stokes@fz-juelich.de

Published by the American Physical Society under the terms of the Creative Commons Attribution 4.0 International license. Further distribution of this work must maintain attribution to the author(s) and the published article's title, journal citation, and DOI. Funded by SCOAP ${ }^{3}$.
}

form factors parameterize interactions where both the initial and final state are the same. To measure them for an (unstable) resonance, one needs to first produce that resonance and then probe it during the extremely short time window before it decays. On the other hand, the transition form factors parameterize the transformation of one state into another. We can probe a stable target such as a ground-state proton and measure how it is excited into the unstable resonance of interest through an examination of its decay products.

It has been suggested that the magnetic dipole moment of the $N^{*}(1535)$ resonance could be measured through the $\gamma p \rightarrow \gamma \eta p$ process [1] using the Crystal Barrel/Two-Arm Photon Spectrometer detector at the Electron Stretcher Accelerator or Crystal Ball at Mainz Microtron, but this measurement has yet to be realized. The difficulty of measuring such quantities experimentally provides the opportunity for lattice QCD to lead experiment and create new knowledge.

\section{A. Structure of excited states}

Here we take the first step and examine the structure of nucleon excitations as observed in the finite volume of lattice QCD. Using local three-quark operators on the lattice, both the Special Research Centre for the Subatomic Structure of Matter (CSSM) [2,3] and the Hadron Spectrum Collaboration (HSC) $[4,5]$ observe two low-lying odd-parity states in the resonance regimes of the $N^{*}(1535)$ and $N^{*}(1650)$.

As these finite-volume states have good overlap with local three-quark operators, we wish to examine the 
extent to which these states, created in relativistic quantum field theory, resemble the quark-model states postulated to describe these resonances $[1,6,7]$. We anticipate the lattice-QCD states excited by such operators to be either quark-model-like states dressed by a meson cloud, similar to the ground-state nucleon, or perhaps bound mesonbaryon molecular states, such as the $\Lambda(1405)$ [8].

In Ref. [9], we presented a method for extracting the form factors of a baryonic state on the lattice using the parity-expanded variational analysis (PEVA) technique and established its effectiveness for accessing the structure of the ground-state nucleon. We now use this method (as summarized in Sec. II) to investigate the structure of the excitations of the proton and neutron observed in the finite volume of lattice QCD.

In this paper we present a determination of the Sachs electric and magnetic form factors for three spin- $1 / 2$ nucleon eigenstates on the lattice. Two of these states are negativeparity nucleon excitations, which we label $N_{1}^{*}$ (or $p_{1}^{*}$ for the proton excitation and $n_{1}^{*}$ for the neutron excitation) and $N_{2}^{*}$ (or the equivalent labels for the excited proton and neutron). The remaining eigenstate is a positive-parity excitation and is denoted $N^{\prime}, p^{\prime}$, or $n^{\prime}$.

We compare the magnetic moments drawn from the negative-parity lattice-QCD results to constituent-quarkmodel predictions for the magnetic moments of the $N^{*}(1535)$ and $N^{*}(1650)$ resonances $[1,6]$. Such quarkmodel calculations can be extended to include effects from the pion cloud. We also compare our lattice results to two such extensions [6,7]. From these comparisons, we make connections to the basis states to be considered in future Hamiltonian effective field theory (HEFT) analyses [10]. Finally, we examine the extent to which the electromagnetic form factors of the positive-parity excitation at $\sim 1900 \mathrm{MeV}$ are consistent with a constituent-quark-model radial excitation of the ground-state nucleon.

\section{B. Toward baryon resonance structure}

In determining resonance properties from lattice-QCD calculations, one requires a comprehensive understanding of the spectrum of excited states in the finite periodic volume of the lattice. This spectrum includes all single, hybrid, and multiparticle contributions having the quantum numbers of the resonance of interest. This finite-volume spectrum composes the input into the Lüscher method [11] or its generalizations $[12,13]$ which relate the finite-volume energy levels to infinite-volume momentum-dependent scattering amplitudes. The application of these methods is a necessary step in connecting lattice QCD to the resonance properties measured in experiment.

Obtaining an accurate determination of the finite-volume nucleon spectrum is challenging. It requires an extensive collection of baryon interpolating fields and robust correlation function analysis techniques. Many collaborations have explored the nucleon spectrum excited by local single- particle operators $[2,4,5,14-19]$. Hybrid nucleon interpolators have been investigated in Ref. [20], where additional states were found in the spectrum. Nonlocal multiparticle interpolating fields are necessary to quantify avoided level crossings and determine the lattice energy eigenstates to the level of accuracy [21] required for the implementation of the Lüscher formalism, bringing lattice-QCD results to experiment. In light of these challenges, the main approach has been to bring experimental measurements to the finite volume of the lattice $[18,22,23]$. It is only recently that the first applications of the Lüscher formalism to the latticebaryon spectrum have emerged [24,25].

The computational challenges in the baryon sector contrast the tremendous progress made within the meson sector. For example, using the formalism for connecting precision finite-volume lattice-QCD matrix-element calculations to the transition amplitudes of experiment [26,27], the resonant $\pi^{+} \gamma \rightarrow \pi^{+} \pi^{0}$ amplitude was first explored in Ref. [28]. More recently, the $\pi \pi \rightarrow \pi \gamma^{\star}$ amplitude [29], the resonant $\rho \rightarrow$ $\pi \gamma^{\star}$ transition [29], the $\pi \gamma \rightarrow \pi \pi$ transition [30] and $\rho$-meson radiative decay [30] have been studied. In these calculations, the finite-volume lattice matrix elements are related to the physical momentum-transfer and energy-dependent scattering observables of experiment. Here a resonance appears as an enhancement as a function of the scattering energy.

A formalism for connecting the finite-volume matrix elements under investigation herein to experiment has been presented in Refs. [31,32]. While our lattice-QCD formalism for matrix-element determination respects the subtleties of the finite volume, the calculations are not sufficiently precise to engage in the connection to experimental scattering observables. There, one needs the contributions of multiparticle scattering states to ensure eigenstate-projected correlation functions contain no contaminations, to quantify the exact eigenstate energies, and to include their contributions to resonances, which can be spread over several finite-volume energy eigenstates, particularly for large lattice volumes where the quantized momentum spacing becomes narrow.

The calculation of lattice matrix elements for momentum-projected meson-baryon interpolators has yet to be reported in the literature. However, their calculation for nonforward momentum transfers will be founded on the formalism presented and utilized herein.

\section{Overview}

In this paper we calculate the Sachs electric and magnetic form factors for three spin- $1 / 2$ nucleon excitations observed on the finite-volume lattice. We commence with a brief summary of the parity-expanded variational analysis for matrix elements in Sec. II. There, the highlights of how the PEVA projectors alter the standard formalism is presented. Lattice-QCD gauge fields, parameters and associated analysis techniques are summarized in Sec. III.

Section IV presents calculations of the electromagnetic form factors for the two low-lying negative-parity 
excitations observed on the lattice. There, the focus is on the utility of the PEVA formalism in removing oppositeparity contaminations from the lattice correlation functions. The importance of the formalism is quantified by comparing with a conventional variational analysis where opposite-parity contaminations are not addressed through an expansion of the correlation matrix. Of particular note is a comparison of the magnetic moments drawn from the negative-parity lattice-QCD results to constituent-quarkmodel predictions in Sec. IV E.

Finally, the electromagnetic structure of the first positiveparity excitation observed at $\sim 1900 \mathrm{MeV}$ in our latticeQCD calculations is presented in Sec. V. The extent to which this excitation is consistent with a constituent-quarkmodel radial excitation of the ground-state nucleon is of particular interest. In accord with other studies [33,34], we find the structure to be consistent with a radial excitation, further strengthening the case that the Roper resonance is not associated with a quark-model-like state $[18,23,35]$.

A summary and outline of future work is provided in the conclusions of Sec. VI.

\section{PARITY-EXPANDED VARIATIONAL ANALYSIS}

The process of extracting finite-momentum matrix elements of baryonic excited states via the PEVA technique is presented in full in Ref. [9]. We provide here a brief summary of this process to introduce the notation and concepts necessary to present our results.

The idea of using operator overlaps to project onto excited states [36-38] and separate opposite parities $[39,40]$ has also been considered in the meson sector. In this case, matrix elements can be extracted using standard techniques [36-38] as the intricacies of parity mixing within the spinor components of the correlator are absent. In the baryon sector, one must take the PEVA projectors into account in identifying the appropriate Dirac-index combinations required to isolate the covariant vertex functions and associated Sachs form factors.

We begin with a basis of $n$ conventional spin- $1 / 2$ operators $\left\{\chi_{i}(x)\right\}$ that couple to the states of interest. Adopting the Pauli representation of the gamma matrices, we introduce the PEVA projector [41] $\Gamma_{ \pm} \equiv \frac{1}{4}\left(\mathbb{I}+\gamma^{4}\right)\left(\mathbb{I} \pm i \gamma^{5} \gamma^{k} \hat{p}^{k}\right)$ and construct a set of basis operators:

$$
\begin{gathered}
\chi_{ \pm p i}(x) \equiv \Gamma_{ \pm p} \chi_{i}(x), \\
\chi_{ \pm p i^{\prime}}(x) \equiv \pm \Gamma_{ \pm p} \gamma^{5} \chi_{i}(x) .
\end{gathered}
$$

We note that we use a Euclidean metric $\delta^{\mu \nu}$, and hence there is no need to distinguish between contravariant and covariant indices.

We then seek an optimized set of operators $\phi_{ \pm p}^{\alpha}(x)$ that each couple strongly to a single energy eigenstate $\alpha$. These optimized operators are constructed as linear combinations of the basis operators. The optimum linear combinations are found by solving a generalized eigenvalue problem with $G(p ; t+\Delta t)$ and $G(p ; t)$, where the correlation matrix

$$
G_{i j}(p ; t) \equiv \operatorname{Tr}\left(\sum_{x} \mathrm{e}^{-i p \cdot x}\left\langle\Omega\left|\chi_{ \pm p i}(x) \bar{\chi}_{ \pm p i}(0)\right| \Omega\right\rangle\right),
$$

with $i$ and $j$ ranging over both the primed and unprimed operators. This process is described in detail in Ref. [41].

Using the optimized operators, we can construct the eigenstate-projected two-point correlation function

$$
\begin{aligned}
G(p ; t ; \alpha) & \equiv \operatorname{Tr}\left(\sum_{x} \mathrm{e}^{-i p \cdot x}\left\langle\Omega\left|\phi_{ \pm p}^{\alpha}(x) \bar{\phi}_{ \pm p}^{\alpha}(0)\right| \Omega\right\rangle\right) \\
& =v_{i}^{\alpha}(p) G_{i j}(p ; t) u_{j}^{\alpha}(p)
\end{aligned}
$$

and the three-point correlation functions

$$
\begin{aligned}
\mathcal{G}_{ \pm}^{3}\left(\mathcal{J} ; p^{\prime}, p ; t_{2}, t_{1} ; \alpha\right) \equiv & \sum_{x_{2}, x_{2}} \mathrm{e}^{-i p^{\prime} \cdot x_{2}} \mathrm{e}^{i\left(p^{\prime}-p\right) \cdot x_{1}} \\
& \times\left\langle\Omega\left|\phi_{ \pm p^{\prime}}^{\alpha}\left(x_{2}\right) \mathcal{J}\left(x_{1}\right) \bar{\phi}_{+p}^{\alpha}(0)\right| \Omega\right\rangle
\end{aligned}
$$

where $\mathcal{J}(x)$ is some current operator, which is inserted with a momentum transfer $q=p^{\prime}-p$. The consideration of $\mathcal{G}_{-}^{3}\left(\mathcal{J} ; p^{\prime}, p ; t_{2}, t_{1} ; \alpha\right)$ (where the sink operator uses the opposite PEVA projector sign convention to the source operator) is required to optimize the extraction of the form factors for general kinematics. We note that it is sufficient to consider this change of projector for the sink operator alone, leaving the source operator as $\bar{\phi}_{+p}^{\alpha}(0)$ in all cases considered.

In this paper, we investigate the electromagnetic properties of the proton and neutron by choosing the current operator $\mathcal{J}(x)$ to be the vector current. In particular, we use the $O(a)$-improved [42] conserved vector current used in Ref. [43]:

$$
j_{C I}^{\mu}(x) \equiv j_{C}^{\mu}(x)+\frac{r}{2} a \bar{q}(x)\left(\overleftarrow{\nabla}^{\rho}+\vec{\nabla}^{\rho}\right) \sigma^{\rho \mu} q(x),
$$

where $r$ is the Wilson parameter and $j_{C}^{\mu}(x)$ is the standard conserved vector current for the Wilson action.

This choice of current operator gives the matrix element

$$
\begin{aligned}
\left\langle\alpha ; p^{\prime} ; s^{\prime}\left|j_{C I}^{\mu}(0)\right| \alpha ; p ; s\right\rangle= & \sqrt{\frac{m^{\alpha}}{E^{\alpha}(p)}} \sqrt{\frac{m^{\alpha}}{E^{\alpha}\left(p^{\prime}\right)}} \bar{u}^{\alpha}\left(p^{\prime}, s^{\prime}\right) \\
& \times\left(\gamma^{\mu} F_{1}^{\alpha}\left(Q^{2}\right)-\frac{\sigma^{\mu \nu} q^{\nu}}{2 m^{\alpha}} F_{2}^{\alpha}\left(Q^{2}\right)\right) \\
& \times u^{\alpha}(p, s),
\end{aligned}
$$

where $Q^{2}=q^{2}-\left(E^{\alpha}\left(p^{\prime}\right)-E^{\alpha}(p)\right)^{2}$ is the squared fourmomentum with the conventional sign, and the invariant 
scalar functions $F_{1}\left(Q^{2}\right)$ and $F_{2}\left(Q^{2}\right)$ are, respectively, the Dirac and Pauli form factors. Here $u^{\alpha}(p, s)$ is the spinor for the lattice eigenstate $\alpha$ moving with momentum $p$ and $\operatorname{spin} s$. As $\alpha$ is an eigenstate of the lattice Hamiltonian, this spinor takes the form of a conventional single-particle spinor with the center-of-momentum energy $E_{C M}=\sqrt{E^{\alpha}(p)^{2}-\vec{p}^{2}}$ playing the role of mass in the finite volume. The states considered in this work display an energy-momentum relation consistent with a single-particle dispersion relation [41]. As such, $E_{C M} \approx m^{\alpha}$ throughout this work. However, the techniques presented respect the subtleties of the finite volume and are applicable for states where $E_{C M} \neq m^{\alpha}$.
To extract our desired signal from this spinor structure, we can take the spinor trace with some spin-structure projector $\Gamma_{S}$. This trace is then called the spinor-projected three-point correlation function

$$
\begin{aligned}
& G_{ \pm}^{3}\left(\Gamma_{S} ; j_{C I}^{\mu} ; p^{\prime}, p ; t_{2}, t_{1} ; \alpha\right) \\
& \quad \equiv \operatorname{Tr}\left(\Gamma_{S} \mathcal{G}_{ \pm}^{3}\left(j_{C I}^{\mu} ; p^{\prime}, p ; t_{2}, t_{1} ; \alpha\right)\right) .
\end{aligned}
$$

These spinor-projected correlation functions have a nontrivial time dependence, which can be removed by constructing the ratio [44]

$$
\begin{aligned}
R_{ \pm}\left(p^{\prime}, p ; \alpha ; r, s\right) \equiv & \sqrt{\left|\frac{r^{\mu} G_{ \pm}^{3}\left(s^{\nu} \Gamma^{\nu} ; j_{C I}^{\mu} ; p^{\prime}, p ; t_{2}, t_{1} ; \alpha\right) r^{\rho} G_{ \pm}^{3}\left(s^{\sigma} \Gamma^{\sigma} ; j_{C I}^{\rho} ; p, p^{\prime} ; t_{2}, t_{1} ; \alpha\right)}{G\left(p^{\prime} ; t_{2} ; \alpha\right) G\left(p ; t_{2} ; \alpha\right)}\right|} \\
& \times \operatorname{sgn}\left(r^{\lambda} G_{ \pm}^{3}\left(s^{\eta} \Gamma^{\eta} ; j_{C I}^{\lambda} ; p^{\prime}, p ; t_{2}, t_{1} ; \alpha\right)\right),
\end{aligned}
$$

where $\Gamma^{4}=\left(\mathbb{I}+\gamma^{4}\right) / 2$ and $\Gamma^{k}=\left(\mathbb{I}+\gamma^{4}\right)\left(i \gamma^{5} \gamma^{k}\right) / 2$ form the basis for the spin projectors we use and $r^{\mu}$ and $s^{\mu}$ are coefficients selected to determine the form factors.

We can then define the reduced ratio

$$
\begin{aligned}
\bar{R}_{ \pm}\left(p^{\prime}, p ; \alpha ; r, s\right) \equiv & \sqrt{\frac{2 E^{\alpha}(p)}{E^{\alpha}(p)+m^{\alpha}}} \sqrt{\frac{2 E^{\alpha}\left(p^{\prime}\right)}{E^{\alpha}\left(p^{\prime}\right)+m^{\alpha}}} \\
& \times R_{ \pm}\left(p^{\prime}, p ; \alpha ; r, s\right) .
\end{aligned}
$$

By investigating the $r^{\mu}$ and $s^{\sigma}$ dependence of this ratio, we find that the clearest signals are given by

$$
\begin{aligned}
& R_{ \pm}^{T}=\frac{2}{1 \pm \hat{p} \cdot \hat{p}^{\prime}} \bar{R}_{ \pm}\left(p^{\prime}, p ; \alpha ;(1,0),(1,0)\right), \\
& R_{\mp}^{S}=\frac{2}{1 \pm \hat{p} \cdot \hat{p}^{\prime}} \bar{R}_{\mp}\left(p^{\prime}, p ; \alpha ;(0, \hat{r}),(0, \hat{s})\right),
\end{aligned}
$$

where $\hat{s}$ is chosen such that $p \cdot \hat{s}=0=p^{\prime} \cdot \hat{s}, \hat{r}$ is equal to $\hat{q} \times \hat{s}$, and the sign \pm in Eq. (10) is chosen such that $1 \pm$ $\hat{p} \cdot \hat{p}^{\prime}$ is maximized. This choice maximizes the signal in the lattice determination of the correlation function ratios.

We can then find the Sachs electric and magnetic form factors

$$
\begin{aligned}
G_{E}^{\alpha}\left(Q^{2}\right) & \equiv F_{1}^{\alpha}\left(Q^{2}\right)-\frac{Q^{2}}{\left(2 m^{\alpha}\right)^{2}} F_{2}^{\alpha}\left(Q^{2}\right), \\
G_{M}^{\alpha}\left(Q^{2}\right) & \equiv F_{1}^{\alpha}\left(Q^{2}\right)+F_{2}^{\alpha}\left(Q^{2}\right),
\end{aligned}
$$

through appropriate linear combinations of $R_{ \pm}^{T}$ and $R_{\mp}^{S}$.

We have shown how the PEVA technique can be applied to the calculation of elastic baryon form factors for arbitrary kinematics. We now proceed to investigate the Sachs electric and magnetic form factors of several excitations of the nucleon.

\section{LATTICE-QCD PARAMETERS AND ANALYSIS TECHNIQUES}

\section{A. Gauge field configurations}

The results presented in this paper are calculated on the PACS-CS $(2+1)$-flavor full-QCD ensembles [45], made available through the International Lattice Data Grid [46]. These ensembles use a $32^{3} \times 64$ lattice and employ a renormalization-group improved Iwasaki gauge action with $\beta=1.90$ and nonperturbatively $O(a)$ improved Wilson quarks, with $C_{S W}=1.715$. We use five ensembles, with stated pion masses from $m_{\pi}=702$ to $156 \mathrm{MeV}$ [45], and set the scale using the Sommer parameter with $r_{0}=0.4921(64) \mathrm{fm}$ [45]. More details of the individual ensembles are presented in Table I, including the squared pion masses in the Sommer scale. When fitting correlators, the $\chi^{2} /$ d.o.f. is calculated with the full covariance matrix, and the $\chi^{2}$ values of all fits are consistent with an appropriate $\chi^{2}$ distribution.

The three heaviest pion masses available among these ensembles span $m_{\pi}=411-702 \mathrm{MeV}$, a typical range for contemporary studies of baryon excitations. As such, these masses are appropriate for this world-first study of the electromagnetic structure of nucleon excitations in lattice QCD. In presenting our discoveries, we will focus on the results at these three heaviest pion masses. There are two lighter masses, at $m_{\pi}=156$ and $296 \mathrm{MeV}$. These approach the physical point, presenting a significant challenge in 
TABLE I. Details of the gauge field ensembles used in this analysis. For each ensemble we list both the pion mass given in Ref. [45], with the lattice spacing set by hadronic inputs, and our determination of the squared pion mass with the lattice spacing listed in the table, which is set by the Sommer parameter with $r_{0}=0.4921(64) \mathrm{fm}$ [45]. We also include information on the statistics used, listing the number of gauge field configurations in each ensemble, and the number of unique quark propagator source locations used on each configuration.

\begin{tabular}{lcccc}
\hline \hline PACS-CS $m_{\pi} / \mathrm{MeV}$ & $a / \mathrm{fm}$ & $m_{\pi}^{2} / \mathrm{GeV}^{2}$ & No. of conf. & No. sources per conf. \\
\hline 702 & $0.1022(15)$ & $0.3884(113)$ & 399 & 1 \\
570 & $0.1009(15)$ & $0.2654(81)$ & 397 & 1 \\
411 & $0.0961(13)$ & $0.1525(43)$ & 449 & 2 \\
296 & $0.0951(13)$ & $0.0784(25)$ & 400 & 2 \\
156 & $0.0933(13)$ & $0.0285(12)$ & 197 & 4 \\
\hline \hline
\end{tabular}

terms of gauge noise and computational cost, but offer the possibility of insight into important chiral physics.

\section{B. Conventional and PEVA techniques}

For the variational analyses in this paper, we begin with the same eight-interpolator basis as in Ref. [9], in which we studied the electromagnetic form factors of the ground-state nucleon. This basis is formed from the conventional spin- $1 / 2$ nucleon interpolators

$$
\begin{aligned}
& \chi_{1}=\epsilon^{a b c}\left[u^{a \top}\left(C \gamma^{5}\right) d^{b}\right] u^{c} \quad \text { and } \\
& \chi_{2}=\epsilon^{a b c}\left[u^{a \top}(C) d^{b}\right] \gamma^{5} u^{c},
\end{aligned}
$$

with $16,35,100$, or 200 sweeps of gauge-invariant Gaussian smearing [47] with a smearing fraction of $\alpha=0.7$, applied at the quark source and sinks in creating the propagators. Before performing the Gaussian smearing, the gauge links to be used are smoothed by applying four sweeps of three-dimensional isotropic stout-link smearing [48] with $\rho=0.1$. We will refer to analyses based on this $8 \times 8$ correlation matrix, without opposite-parity interpolators, as the conventional variational analysis. For the PEVA technique, this basis is expanded to 16 operators as described in Sec. II.

We study the first three excitations extracted by this basis, consisting of one positive-parity state and two negative-parity states. As we will see in the results presented below, the PEVA technique is very important in correctly extracting form factors of these excitations.

\section{Three-point function techniques}

To extract the form factors, we fix the source at time slice $N_{t} / 4=16$ relative to a fixed boundary condition in time, and (utilizing the sequential source technique [49]) invert through the current, fixing the current insertion at time slice 21 . We choose time slice 21 by inspecting the two-point correlation functions associated with each state and observing that excited-state contaminations in the eigenstateprojected correlators are suppressed by time slice 21 . This is evaluated by fitting the effective mass in this region to a single-state ansatz verifying that the full covariant $\chi^{2} /$ d.o.f. is satisfactory. We then extract the form factors as outlined in Sec. II for every possible sink time and once again look for a plateau consistent with a single-state ansatz.

\section{Multiparticle scattering-state contributions}

The quasilocal operators used to excite the states of interest do not have good overlap with multiparticle scattering eigenstates. As such, a particular concern in this analysis is the possibility of contamination of our correlation functions by nearby multiparticle scattering states that have not been isolated in the current correlation-matrix analysis using local operators.

Fortunately, significant mixing of one- and two-particle basis states gives rise to avoided level crossings creating a large energy separation between the lattice energy eigenstates. This difference in energies leads to a rejection of single-state ansatz fits [16] signified by a large covariancematrix $\chi^{2} /$ d.o.f.

However, when the mixing of the basis states is small, the avoided level crossing effects are significantly reduced. This can allow lattice eigenstates with closely spaced energy levels. Such nearby eigenstates are more problematic as simple Euclidean time evolution cannot expose separate states. If one is interested only in the energies of the eigenstates, the mixing can shift the observed energies by an small amount, typically within the width of the associated resonance. On the other hand, it is these subtle shifts that are central to the Lüscher formalism [21]. This issue of subtle state mixing applies to form factors in a more significant manner as the form factors of the scattering states may differ significantly from the energy eigenstates having good overlap with the local interpolators. Thus it is important to estimate the extent of this mixing.

For the two low-lying negative-parity states, an examination of the two-particle threshold energies relative to the energies of the observed excitations can provide some insight. Table II presents Sommer-scale masses and twoparticle infinite-volume threshold energies which can be compared with the masses of the first and second negativeparity states observed on the lattice [10]. While one does not have any insight into how the $\pi N$ threshold energy gets 
TABLE II. Sommer-scale masses and two-particle infinitevolume threshold energies in units of $\mathrm{GeV}$ are compared with the masses of the first, $N_{1}^{*}$, and second, $N_{2}^{*}$, negative-parity states observed on the lattice.

\begin{tabular}{ccccc}
\hline \hline$m_{\pi} / \mathrm{GeV}$ & $m^{N} / \mathrm{GeV}$ & $\left(m^{N}+m_{\pi}\right) / \mathrm{GeV}$ & $m^{N_{1}^{*}} / \mathrm{GeV}$ & $m^{N_{2}^{*}} / \mathrm{GeV}$ \\
\hline $0.623(9)$ & $1.41(1)$ & $2.03(1)$ & $1.90(4)$ & $1.95(2)$ \\
$0.515(8)$ & $1.27(1)$ & $1.78(1)$ & $1.78(5)$ & $1.82(2)$ \\
$0.391(6)$ & $1.15(1)$ & $1.54(1)$ & $1.71(3)$ & $1.77(3)$ \\
$0.280(4)$ & $1.06(1)$ & $1.34(1)$ & $1.56(5)$ & $1.75(9)$ \\
$0.169(4)$ & $1.01(4)$ & $1.18(4)$ & $1.49(12)$ & $1.55(8)$ \\
\hline \hline
\end{tabular}

dressed in the finite volume relative to $N_{1}^{*}$ and $N_{2}^{*}$ negativeparity states, one can see that at the largest quark mass considered, the threshold is above the two energies observed on the lattice. In light of the volume suppression of two-particle couplings to local interpolating fields, scattering-state contamination is not a significant concern at this heaviest quark mass.

At the second heaviest mass considered, the situation is more complicated and one must turn to calculations that do account for the mixing of the $\pi N$ threshold basis state with other states in the system. HEFT calculations for odd-parity nucleon excitations can provide considerable insight $[10,50]$. Figures 2 and 3 of Ref. [50] indicate the $\pi N$ basis state is strongly mixed in creating the two lowest-lying oddparity states observed on the lattice. Once again, we observe that there is no low-lying $\pi N$ scattering-state contaminant.

At the middle quark mass considered, the $\pi N$ scattering state lies well below the two states seen in lattice QCD. Here, HEFT can provide some insight if one uses the overlap of the local bare basis state with the energy eigenstates in HEFT as a proxy for the overlap of the local lattice interpolating fields with the lattice energy eigenstates. The idea is that the bare basis state in HEFT is the only localized basis state in the theory. Drawing on chiral perturbation theory, one can show the overlap of a smeared interpolating field with nonlocal momentum-projected $\pi N$ basis states is suppressed by factor of $\sim 10^{-3}$ relative to the ground state [51] for our current lattice parameters. Thus, the optimized smeared interpolating field of lattice QCD is associated with the bare basis state of HEFT, $\left|m_{0}\right\rangle$, and the element $\left|\left\langle m_{0} \mid E_{\alpha}\right\rangle\right|^{2}$ of the HEFT eigenvector governs the relative probability of exciting eigenstate $\left|E_{\alpha}\right\rangle$. With this approximation, Fig. 3 of Ref. [50] suggests a 5\% scatteringstate contribution to our projected correlators.

At the second lightest of the quark masses considered herein, the mixing of the low-lying two-particle $\pi N$ scattering state is large enough to be quantified [52]. While no scattering-state contamination is manifest in the eigenstate-projected correlator associated with the $N^{*}(1535)$, the correlator associated with the second negative-parity excitation did reveal a small contamination. By extending the Euclidean-time fit regime into the tail of the projected two-point correlator, the high- precision analysis of Ref. [52] resolves a second lowlying state consistent with a $\pi N$ scattering state at the $10 \%$ level. This contribution is consistent with expectations from HEFT [50]. Our present calculation avoids the tail of this projected correlator and our use of the single-state ansatz [16] ensures that these contributions to the effective energy are contained within the statistical uncertainties of the results. However, as shown in Ref. [9], contaminants that do not significantly perturb the extracted mass can still have a significant effect on the extracted form factors. As such, we must be cautious when interpreting results from this state at this mass.

Finally, at the lightest quark mass considered, we anticipate a similar contribution from $\pi N$ scattering states. However, statistical uncertainties at the lightest quark mass are large and we have been unable to resolve any evidence of scattering-state contamination. As one moves toward a precise examination of these states, one must also accommodate $K \Lambda$ and $K \Sigma$ scattering states in the analysis as the energies of these scattering-state thresholds are in the regime of the $N^{*}$ states under examination at this nearphysical quark mass.

With regard to the positive-parity excitation examined in Sec. V, the formidable challenge of extracting full knowledge of the many possible scattering-state contributions to the spectrum of eigenstates on the lattice is well beyond the current capabilities of the lattice community and may only be realized with the benefit of significant algorithmic and/ or computational advances. For example, at the lightest quark mass considered, there is a multiparticle scattering state associated with a nucleon plus five pions which lies below the first excitation observed on the lattice at $\simeq 1.9 \mathrm{GeV}$. Again, using the overlap of the bare basis state with the energy eigenstates in HEFT as a proxy for the overlap of the local lattice interpolators with the lattice energy eigenstates, Figs. 3 and 5 of Ref. [23] indicate the only states having significant overlap with local interpolating fields are the states under examination herein.

This expectation is in accord with the results of Ref. [19] based on the same PACS-CS lattices examined herein. There Fig. 4 illustrates how the inclusion of low-lying momentum-projected two-particle $\pi N$ and $\sigma N$ interpolators has a marginal effect on the mass of the state determined with local interpolators alone.

In summary, the contamination of our correlation functions by nearby multiparticle scattering states that have not been isolated in the current correlation-matrix analysis using local operators is expected to be small. At the heaviest quark masses, there is no issue with low-lying scattering states. At the lightest quark masses, a small contamination of approximately $10 \%$ may be found in the projected correlator of the second negative-parity excitation most associated with the $N^{*}(1650)$ resonance. As our main focus is on the heaviest three masses and quark-model comparisons, scattering-state contributions do not pose a 
significant issue in this first examination of excited-state electromagnetic structure. However, future calculations seeking a quantitative connection to the scattering observables of experiment will require the inclusion of nonlocal multiparticle interpolating fields.

\section{NEGATIVE-PARITY EXCITATIONS}

\section{A. $G_{E}$ for the first negative-parity excitation}

\section{Quark-flavor contributions}

Beginning with the lowest-lying negative-parity excitation observed in this study, we examine how extractions of $G_{E}\left(Q^{2}\right)$ by both the PEVA technique and the conventional analysis defined in Sec. III B depend on the Euclidean time of the sink. In Fig. 1, we plot the connected contributions to $G_{E}\left(Q^{2}\right)$ from single quarks of unit charge for both quark flavors present in the nucleon interpolator. This plot is at the heaviest quark mass considered with $m_{\pi}=702 \mathrm{MeV}$ and the lowest-momentum kinematics of $p=(0,0,0)$ and $p^{\prime}=(1,0,0)$.

We see that the conventional extraction sits well below the PEVA extraction for all time slices between the current insertion and the point at which the signal is lost to noise. The conventional extraction also has a more significant time dependence than the PEVA extraction, forcing the conventional fit one time slice later. Both of these effects indicate that the conventional analysis is affected by opposite-parity contaminations, which are having a

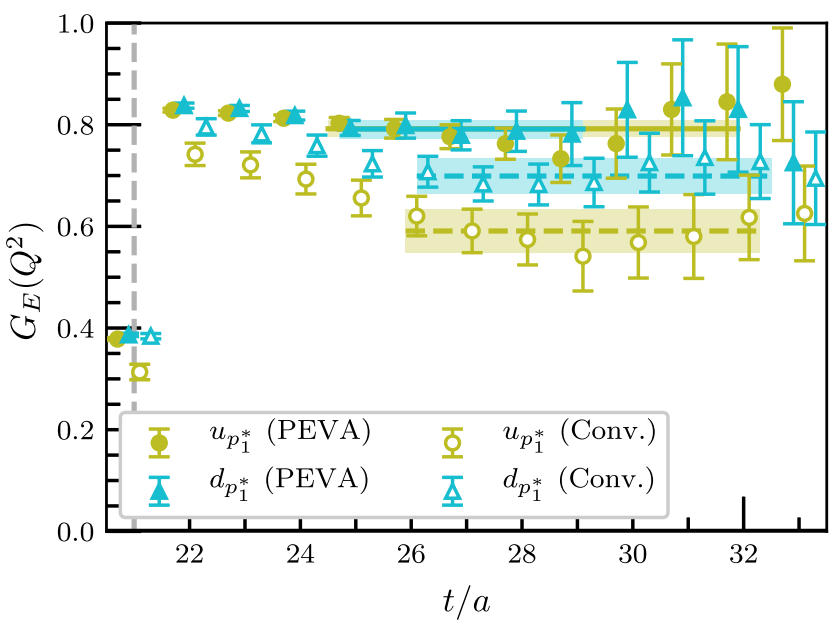

FIG. 1. Quark-flavor contributions to the electric form factor for the first negative-parity excitation of the nucleon at $m_{\pi}=$ $702 \mathrm{MeV}$ for the lowest-momentum kinematics, providing $Q^{2}=0.1424(41) \mathrm{GeV}^{2}$. We plot the conventional analysis with open markers and the new PEVA technique with filled markers. Our fits to the plateaus are illustrated by shaded bands, with dashed fit lines for the conventional analysis and solid fit lines for PEVA. The source is at time slice 16, and the current is inserted at time slice 21, as indicated by the vertical dashed line. Both PEVA fits are from time slice 25, whereas the conventional fits both start at 26 and have significantly lower values than the PEVA fits. significant effect on the extracted form factor, introducing a systematic error of $12(4) \%$ for the singly represented quark flavor and 25(5)\% for the doubly represented flavor.

The lighter pion masses show a similar behavior. The conventional analysis consistently has a plateau which starts later than the PEVA approach and sits significantly lower. For example, at $m_{\pi}=411 \mathrm{MeV}$, the magnitudes of the conventional plateaus with these low-momentum kinematics are systematically underestimated by $19(6) \%$ and $26(6) \%$ for the singly and doubly represented quark flavors, respectively.

We can also consider changing the momenta to access different kinematics. By boosting the initial and final states while keeping the momentum transfer constant, we can access smaller values of $Q^{2}$. We can also increase the threemomentum of the current insertion, giving access to larger values of $Q^{2}$. For such kinematics at all masses we find that, in general, the conventional plateaus are later in time and take smaller values than the PEVA plateaus.

These results indicate that the PEVA technique is critical to the correct extraction of the electric form factors of this nucleon excitation. The conventional analysis is contaminated by opposite-parity states, and when these states are removed by the PEVA technique it has a significant effect on the extracted form factor values. Hence, we now focus our attention only on the PEVA results for the remainder of this subsection.

In Fig. 2, we plot the $Q^{2}$ dependence of the electric form factor at $m_{\pi}=411 \mathrm{MeV}$. The set of kinematics used to access the various $Q^{2}$ values is listed in Table III, and we exclude any fits for which there is no acceptable plateau or the variational analysis fails. We see that the two quark flavors have very similar contributions to the electric form factor. They both agree well with a dipole ansatz

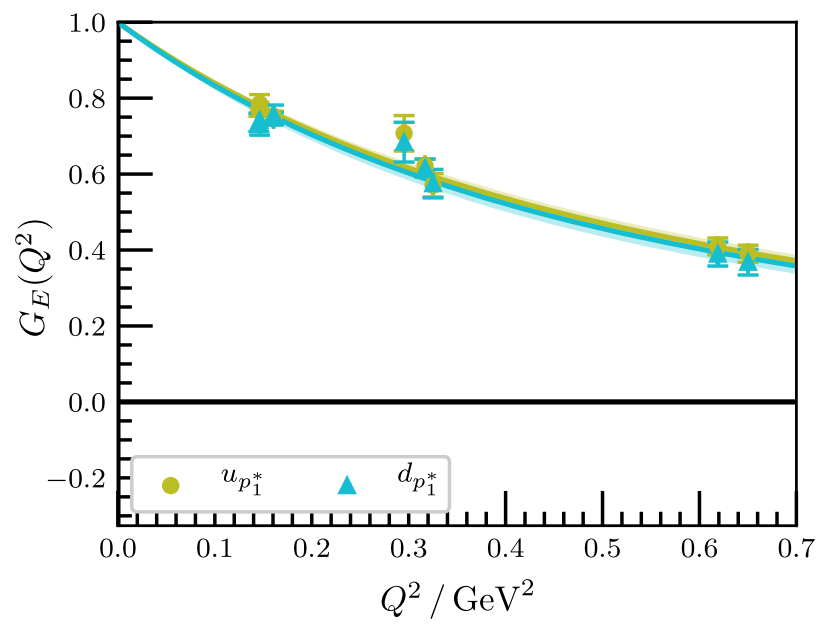

FIG. 2. Quark-flavor contributions to the electric form factor for the first negative-parity excitation at $m_{\pi}=411 \mathrm{MeV}$. The curves are dipole fits to the form factors, with the $y$ intercept fixed to unity. They correspond to rms charge radii of $0.654(20) \mathrm{fm}$ for the doubly represented quark flavor $\left(u_{p_{1}^{*}}\right)$ and $0.670(26)$ fm for the singly represented quark flavor $\left(d_{p_{1}^{*}}\right)$. 
TABLE III. Different kinematics used in our analysis to access a range of $Q^{2}$ values. The $Q^{2}$ value listed is for the first negative-parity nucleon excitation at the middle pion mass of $m_{\pi}=411 \mathrm{MeV}$. The statistical error listed for $Q^{2}$ comes from both the determination of the mass of the state and the conversion to physical units. In the so-called Breit frame kinematics, where the incoming and outgoing energies are equal, the correlated statistical errors from the mass cancel exactly, and as such the only source of errors is uncertainty in the lattice spacing used in converting to physical units.

\begin{tabular}{lcrr}
\hline \hline Source momentum $p$ & Sink momentum $p^{\prime}$ & Momentum transfer $q$ & $Q^{2} / \mathrm{GeV}^{2}$ \\
\hline$(2,0,0)$ & $(3,0,0)$ & $(1,0,0)$ & $0.1224(35)$ \\
$(2,0,1)$ & $(3,0,1)$ & $(1,0,0)$ & $0.1239(35)$ \\
$(1,0,0)$ & $(2,0,0)$ & $(1,0,0)$ & $0.1454(40)$ \\
$(1,0,1)$ & $(2,0,1)$ & $(1,0,0)$ & $0.1462(40)$ \\
$(0,0,0)$ & $(1,0,0)$ & $(1,0,0)$ & $0.1604(44)$ \\
$(0,0,1)$ & $(1,0,1)$ & $(1,0,0)$ & $0.1606(44)$ \\
$(2,0,0)$ & $(3,1,0)$ & $(1,1,0)$ & $0.2683(74)$ \\
$(1,0,0)$ & $(2,1,0)$ & $(1,1,0)$ & $0.2953(81)$ \\
$(0,0,0)$ & $(1,1,0)$ & $(1,1,0)$ & $0.3169(86)$ \\
$(0,-1,0)$ & $(1,0,0)$ & $(2,0,0)$ & $0.3251(89)$ \\
$(1,0,0)$ & $(3,0,0)$ & $(2,0,0)$ & $0.5404(150)$ \\
$(0,0,0)$ & $(2,0,0)$ & $(2,0,0)$ & $0.6190(169)$ \\
$(-1,0,0)$ & $(1,0,0)$ & & $0.6502(177)$ \\
\hline \hline
\end{tabular}

$$
G_{D}\left(Q^{2}\right)=\frac{G_{0}}{\left(1+Q^{2} / \Lambda^{2}\right)^{2}},
$$

with $G_{0}$ fixed to one, as we are working with single quarks of unit charge. These fits correspond to a rms charge radius of $0.654(20)$ fm for the doubly represented quark flavor and $0.670(26) \mathrm{fm}$ for the singly represented quark flavor. These charge radii are similar to the charge radii of the individual quark sectors in the ground state examined in Ref. [9], $[0.662(12) \mathrm{fm}$ for the doubly represented quark flavor and $0.633(12) \mathrm{fm}$ for the singly represented quark flavor]. The doubly represented quark sector agrees to within one standard deviation. However, the singly represented quark sector in the excitation has a charge radius approximately 1.5 standard deviations larger than the ground state.

We see similar behavior for the other four masses. In all cases, the quark distributions are much smaller than the lattice length $L \sim 3 \mathrm{fm}$. The plots for these masses are omitted from this paper for the sake of brevity.

\section{Constituent-quark-model expectations}

Within the context of a simple constituent quark model, the near equivalence of the electric charge radii of quark sectors within the ground-state nucleon and the first negative-parity excitation seems truly remarkable. Considering the effective potential of the radial Schrödinger equation, one expects the repulsive centripetal term proportional to $\ell(\ell+1)$ to force the quarks to larger radii for odd-parity $\ell=1$ states.

However, one needs to recall that these radii are from quantum field theory where dynamical quark-antiquark pairs enable the creation of meson-nucleon components in the $N^{*}$ states. The meson provides the negative parity such that all quarks can reside in relative $s$ waves within the hadrons, forming an $S$-wave meson-baryon molecule. In this way the centripetal barrier is avoided and the negative-parity states can have a size similar to the ground-state nucleon. Relevant meson-baryon channels for the odd-parity states include $K \Sigma$ and $K \Lambda$ in addition to the standard $\eta N$ and $\pi N$ meson-baryon channels.

\section{Baryon electric form factors}

In order to compute the form factors of the first negativeparity excitation of the proton, $G_{E}^{p_{1}^{*}}\left(Q^{2}\right)$, and neutron, $G_{E}^{n_{1}^{*}}\left(Q^{2}\right)$, we need to take the correct linear combinations of the contributions from the doubly represented quark flavor and the singly represented quark flavor to reintroduce the multiplicity of the doubly represented quark and the physical charges of the up and down quarks. To this end we define

$$
\begin{aligned}
& G_{E}^{p_{1}^{*}}\left(Q^{2}\right) \equiv+\frac{4}{3} G_{E}^{u_{p_{1}^{*}}^{*}}\left(Q^{2}\right)-\frac{1}{3} G_{E}^{d_{p_{1}^{*}}}\left(Q^{2}\right), \\
& G_{E}^{n_{1}^{*}}\left(Q^{2}\right) \equiv-\frac{2}{3} G_{E}^{u_{p_{1}^{*}}^{*}}\left(Q^{2}\right)+\frac{2}{3} G_{E}^{d_{p_{1}^{*}}}\left(Q^{2}\right) .
\end{aligned}
$$

In Fig. 3, we plot the nucleon electric form factors obtained by taking these combinations of the form factors at $m_{\pi}=411 \mathrm{MeV}$. The form factor for the neutron excitation is close to zero, reflecting the similar charge radii of the individual quark flavors. By combining the dipole fits to the individual quark sectors in the same way as the data points one obtains a model for the $Q^{2}$ dependence of the electric form factors of the excited proton and neutron that includes full information from both quark sectors. If we do this for all five pion masses, we extract 


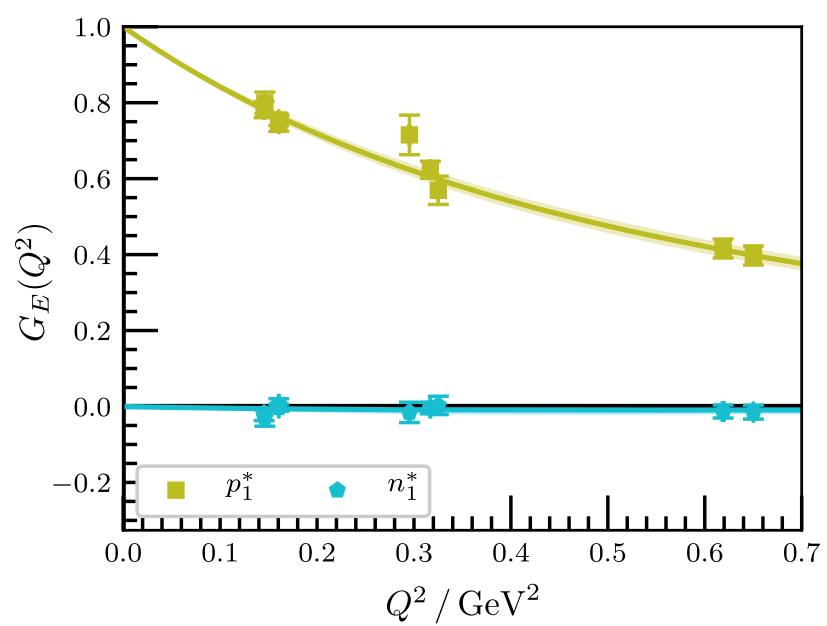

FIG. 3. $G_{E}\left(Q^{2}\right)$ for the first negative-parity excitations of the proton and neutron at $m_{\pi}=411 \mathrm{MeV}$. The curves correspond to linear combinations of the dipole fits to the individual quark sectors from Fig. 2. These combinations provide a squared charge radius of $0.421(29) \mathrm{fm}^{2}$ for the proton and $0.014(18) \mathrm{fm}^{2}$ for the neutron.

squared charge radii for the proton excitation ranging from $0.340(29)$ to $0.470(54) \mathrm{fm}^{2}$, increasing with decreasing pion mass. For the neutron excitation, the squared charge radii are close to or slightly below zero, for example $-0.033(24) \mathrm{fm}^{2}$ at $m_{\pi}=296 \mathrm{MeV}$.

As illustrated in Fig. 4, the pion-mass dependence is fairly smooth and has a clear trend to larger radii at lower pion masses. There is no hint of significant nonanalytic behavior in the quark-mass dependence, due to finitevolume suppression $[53,54]$.

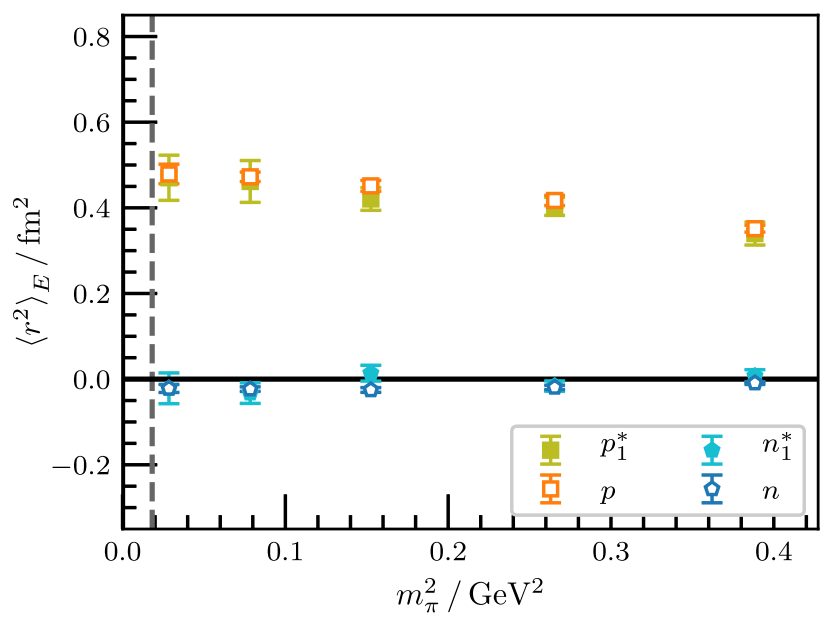

FIG. 4. Quark-mass dependence of squared charge radii for the first negative-parity excitation of the proton and neutron. Results are obtained from dipole fits to the electric form factors of the individual quark sectors. For comparison, the radii for the ground states are plotted with open points. We see a clear trend to larger charge radii as the pion mass approaches the physical pion, represented by the dashed vertical line.
For each pion mass considered, the extracted squared charge radii for this first negative-parity excitation are consistent with the radii of the ground-state proton and neutron at the same mass, as obtained in Ref. [9].

As discussed in the context of the quark-sector contributions, meson-nucleon components in the wave function enable all quarks to reside in relative $s$ waves within the hadrons, forming an $S$-wave meson-nucleon molecule. In this way, the negative-parity states can have a size similar to the ground-state nucleon.

\section{B. $G_{M}$ for first negative-parity excitation}

\section{Quark-flavor contributions}

We now proceed to the magnetic form factor. In Fig. 5, we plot the plateaus at $m_{\pi}=411 \mathrm{MeV}$ with the lowestmomentum kinematics. Here we present results in terms of nuclear magnetons, $\mu_{N} \equiv \frac{e \hbar}{2 m^{\text {hys } p}}$, defined in terms of the physical proton mass, $m^{\text {phys } p}$. While the conventional and PEVA plateaus for the doubly represented quark flavor are consistent, both in fit region and value, the conventional plateau for the singly represented quark flavor starts later and has a significantly more negative value than the PEVA plateau. We see a similar effect at all five pion masses and a variety of kinematics.

Having fit the form factor plateaus, we can investigate the $Q^{2}$ dependence of $G_{M}\left(Q^{2}\right)$. In Fig. 6, we plot the contributions to $G_{M}\left(Q^{2}\right)$ from both the singly represented quark flavor and the doubly represented quark flavor at

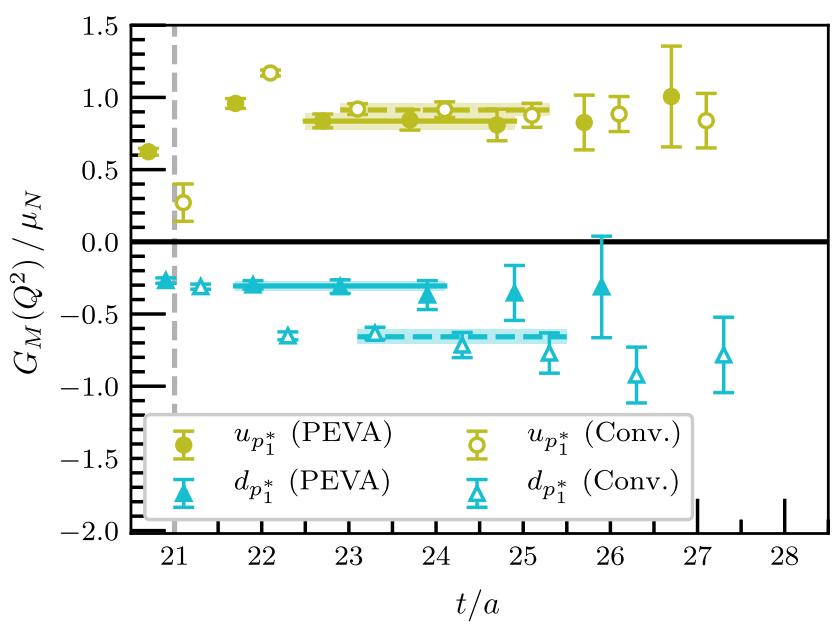

FIG. 5. Quark-flavor contributions to $G_{M}\left(Q^{2}\right)$ for the first negative-parity excitation at $m_{\pi}=411 \mathrm{MeV}$ for the lowestmomentum kinematics, providing $Q^{2}=0.1604(44) \mathrm{GeV}^{2}$. As in Fig. 1, we plot the conventional analysis with open markers and dashed fit lines and the new PEVA approach with filled markers and solid fit lines. For the doubly represented quark flavor, the plateaus for both analyses are from 23 to 25 and take consistent values. For the singly represented quark flavor, the PEVA fit is from 22 to 24 , while the conventional fit is from 23 to 25 , and has a significantly more negative value. 


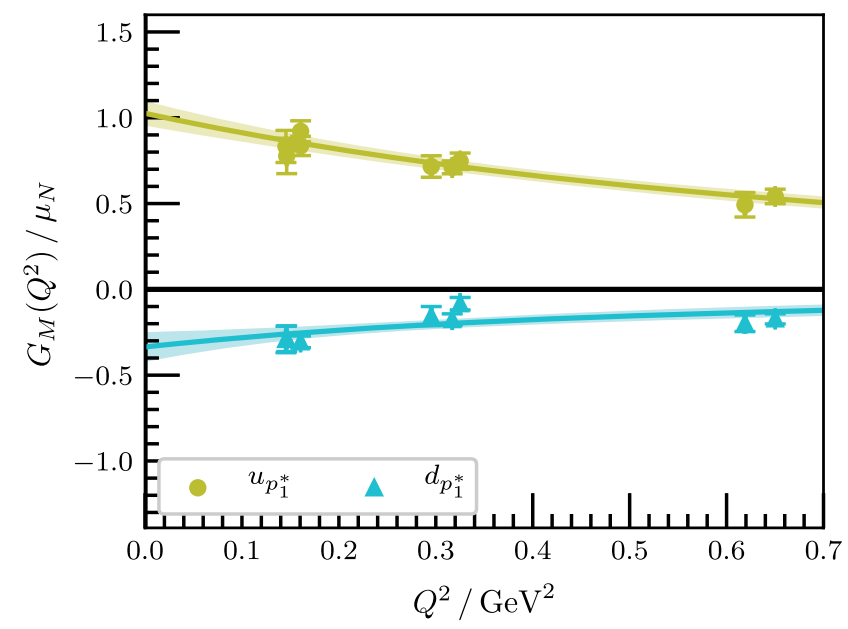

FIG. 6. Quark-flavor contributions to $G_{M}\left(Q^{2}\right)$ for the first negative-parity excitation at $m_{\pi}=411 \mathrm{MeV}$. The curves are dipole fits to the individual quark sectors.

$m_{\pi}=411 \mathrm{MeV}$. Both quark flavors are consistent with a dipole fit. The $Q^{2}$ dependence is similar to that for $G_{E}\left(Q^{2}\right)$, and the same is true for the other pion masses considered.

\section{Baryon magnetic form factors}

As described in Sec. IVA, we can take linear combinations of the individual quark-flavor contributions to compute the magnetic form factors of the excited proton and neutron. We plot these combinations for $m_{\pi}=411 \mathrm{MeV}$ in Fig. 7. The squared magnetic radii given by

$$
\frac{\left\langle r^{2}\right\rangle_{M}}{\mu}=\left.\frac{-6}{G_{M}(0)} \frac{d G_{M}\left(Q^{2}\right)}{d Q^{2}}\right|_{Q^{2}=0}
$$

are obtained via dipole fits to the quark-sector contributions, which allow the value and slope of $G_{M}\left(Q^{2}\right)$ to be

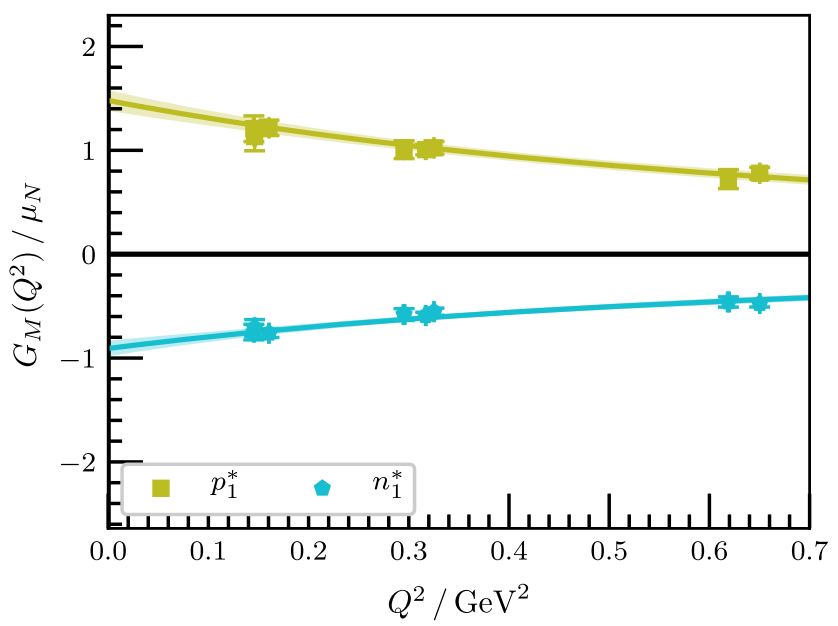

FIG. 7. $G_{M}\left(Q^{2}\right)$ for the first negative-parity excitations of the proton and neutron at $m_{\pi}=411 \mathrm{MeV}$. The curves correspond to linear combinations of the quark-sector dipole fits from Fig. 6. extrapolated to $Q^{2}=0$. In obtaining hadronic magnetic radii, the quark sectors combine with additional weightings given by $G_{M}(0)$, that is,

$$
\begin{aligned}
\frac{\left\langle r^{2}\right\rangle_{M}^{p_{1}^{*}}}{\mu^{p_{1}^{*}}} \equiv & \frac{1}{+\frac{4}{3} G_{M}^{u_{p_{1}^{*}}^{*}}(0)-\frac{1}{3} G_{M}^{d_{p_{1}^{*}}^{*}}(0)} \\
& \times\left(+\frac{4}{3} G_{M}^{u_{p_{1}^{*}}^{*}}(0) \frac{\left\langle r^{2}\right\rangle_{M}^{u_{p_{1}^{*}}^{*}}}{\mu^{u_{p_{1}^{*}}^{*}}}-\frac{1}{3} G_{M}^{d_{p_{1}^{*}}^{*}}(0) \frac{\left\langle r^{2}\right\rangle_{M}^{d_{p_{1}^{*}}}}{\mu_{p_{1}^{*}}}\right), \\
\frac{\left\langle r^{2}\right\rangle_{M}^{n_{1}^{*}}}{\mu^{n_{1}^{*}}} \equiv & \frac{1}{-\frac{2}{3} G_{M}^{u_{p_{1}^{*}}^{*}}(0)+\frac{2}{3} G_{M}^{d_{p_{1}^{*}}^{*}}(0)} \\
& \times\left(-\frac{2}{3} G_{M}^{u_{p_{1}^{*}}^{*}}(0) \frac{\left\langle r^{2}\right\rangle_{M}^{u_{p_{1}^{*}}}}{\mu^{u_{p_{1}^{*}}^{*}}}+\frac{2}{3} G_{M}^{d_{p_{1}^{*}}}(0) \frac{\left\langle r^{2}\right\rangle_{M}^{d_{p_{1}^{*}}^{*}}}{\mu^{d_{p_{1}^{*}}^{*}}}\right) .
\end{aligned}
$$

For all five masses, we find that these squared magnetic radii mostly agree with the charge radii from $G_{E}\left(Q^{2}\right)$.

In Fig. 8, we plot the pion-mass dependence of the squared magnetic radius obtained from quark-sector dipole fits to $G_{M}\left(Q^{2}\right)$ for the excited proton and neutron.

\section{Baryon magnetic moments}

Returning to the individual quark-sector results, we note that $G_{M}\left(Q^{2}\right)$ and $G_{E}\left(Q^{2}\right)$ have a similar $Q^{2}$ dependence over the range considered. In light of this, we hypothesize that $G_{M}\left(Q^{2}\right)$ and $G_{E}\left(Q^{2}\right)$ have the same $Q^{2}$ scaling in this region. If this hypothesis is valid, then the ratio of $G_{M}\left(Q^{2}\right)$ to $G_{E}\left(Q^{2}\right)$ should be independent of $Q^{2}$. Since we are working with an improved conserved vector current, and

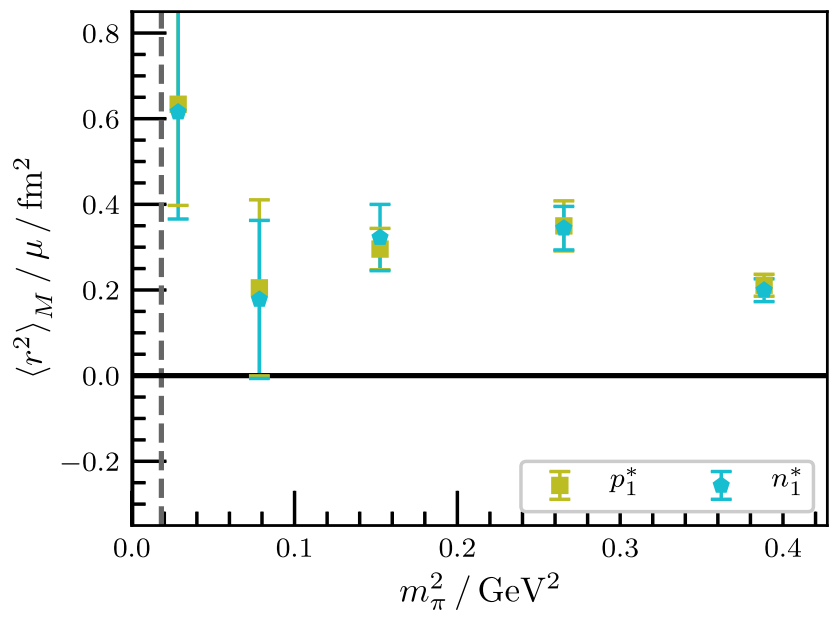

FIG. 8. Quark-mass dependence of squared magnetic radii for the first negative-parity excitation of the proton. 


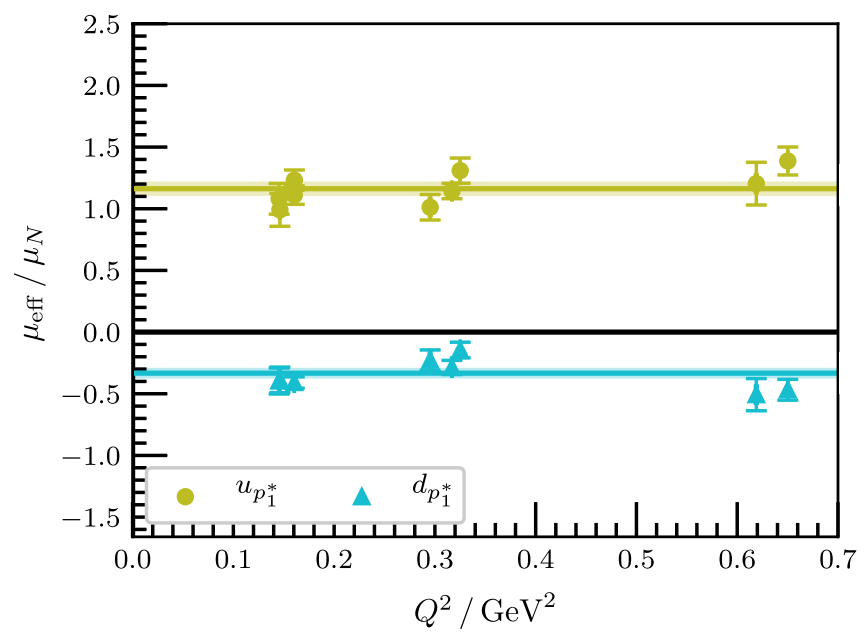

FIG. 9. $\mu_{\text {eff }}$ for individual quarks of unit charge in the first negative-parity nucleon excitation at $m_{\pi}=411 \mathrm{MeV}$. The shaded bands are constant fits to the effective magnetic moment, corresponding to magnetic moment contributions of $1.163(60) \mu_{N}$ for the doubly represented quark and $-0.333(43) \mu_{N}$ for the singly represented quark.

single quarks of unit charge, $G_{E}(0)=1$ exactly, and $G_{M}(0)$ is the contribution of the quark flavor to the magnetic moment (up to scaling by the physical charge). Hence, the ratio

$$
\mu_{\mathrm{eff}}\left(Q^{2}\right) \equiv \frac{G_{M}\left(Q^{2}\right)}{G_{E}\left(Q^{2}\right)}
$$

is expected to provide a measure of the contribution to the magnetic moment from the given quark flavor.

In Fig. 9, we plot this ratio at $m_{\pi}=411 \mathrm{MeV}$ as a function of $Q^{2}$. We see that as expected, the ratio is approximately constant across the $Q^{2}$ range accessible by our kinematics. This holds true for all five pion masses considered in this work. This supports the underlying hypothesis that the $Q^{2}$ scaling of the contributions to $G_{E}\left(Q^{2}\right)$ and $G_{M}\left(Q^{2}\right)$ from each quark sector is the same and, hence, suggests that $\mu_{\text {eff }}$ is a good estimate for the magnetic moment of this state.

We take constant fits to $\mu_{\text {eff }}$ at each quark mass and plot their pion-mass dependence in Fig. 10. By taking linear combinations of these fits as described for $G_{E}\left(Q^{2}\right)$ and $G_{M}\left(Q^{2}\right)$ above, we obtain magnetic moment estimates for the excited proton and neutron, as plotted in Fig. 11. For the heaviest three pion masses, the effective magnetic moments show little pion-mass dependence and have tight error bars. The lightest two pion masses have much larger errors, and we observe a discontinuity in $G_{M}\left(Q^{2}\right)$ at the second lightest mass, appearing as a significantly smaller magnetic moment for both states. This suggests that there could be a change in the structure of this state at that mass. However, there is no corresponding change in $G_{E}\left(Q^{2}\right)$. At the lightest

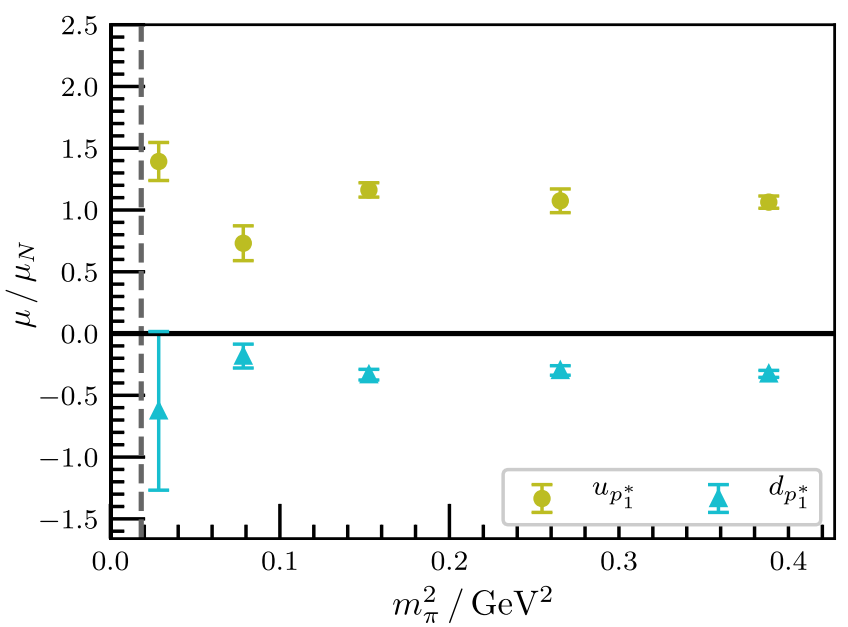

FIG. 10. Quark-mass dependence of contributions from individual unit-charge quarks to the magnetic moment of the first negative-parity nucleon excitation. The vertical dashed line corresponds to the physical pion mass.

mass, the magnetic moments appear to return to consistency with the values from the heavier masses. Hence, it is unclear whether the behavior at the second lightest mass indicates a change in the nature of the state, the presence of significant scattering-state contamination, or is a result of increasing gauge noise at lighter pion masses. Further research will be required to determine which of these possibilities is realized.

In this section focusing on the first negative-parity excitation of the nucleon, we have demonstrated the importance of the PEVA technique in correctly extracting both the electric and magnetic form factors. From these, we derived charge radii, magnetic moments and magnetic radii.

While we regard the results for the three heaviest quark masses to be robust, we take some caution with the lightest

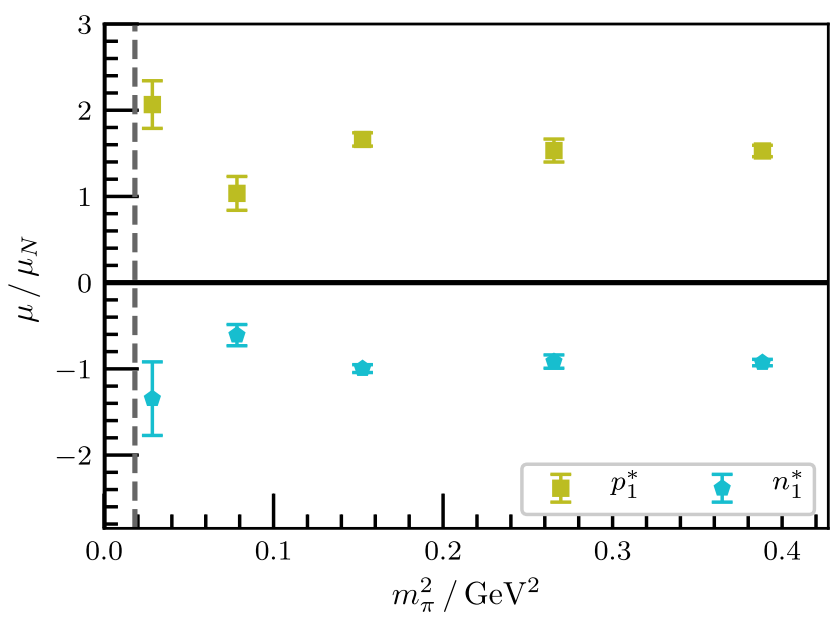

FIG. 11. Quark-mass dependence of the magnetic moment of the first negative-parity excitations of the proton and neutron. 
TABLE IV. Radii and magnetic moments of the ground-state proton and neutron. Radii are obtained from combinations of quark-sector dipole fits and magnetic moments are obtained from quark-sector ratios of $G_{M}\left(Q^{2}\right)$ to $G_{E}\left(Q^{2}\right)$.

\begin{tabular}{lccc}
\hline \hline$m_{\pi}^{2} / \mathrm{GeV}^{2}$ & $\left\langle r^{2}\right\rangle_{E}^{p} / \mathrm{fm}^{2}$ & $\left\langle r^{2}\right\rangle_{M}^{p} / \mu^{p} / \mathrm{fm}^{2}$ & $\mu^{p} / \mu_{N}$ \\
\hline $0.3884(113)$ & $0.351(13)$ & $0.301(13)$ & $1.89(3)$ \\
$0.2654(81)$ & $0.417(17)$ & $0.341(15)$ & $2.10(4)$ \\
$0.1525(43)$ & $0.451(18)$ & $0.340(13)$ & $2.24(4)$ \\
$0.0784(25)$ & $0.472(17)$ & $0.360(16)$ & $2.30(4)$ \\
$0.0285(12)$ & $0.479(27)$ & $0.324(36)$ & $2.52(8)$ \\
\hline \hline$m_{\pi}^{2} / \mathrm{GeV}^{2}$ & $\left\langle r^{2}\right\rangle_{E}^{n} / \mathrm{fm}^{2}$ & $\left\langle r^{2}\right\rangle_{M}^{n} / \mu^{n} / \mathrm{fm}^{2}$ & $\mu^{n} / \mu_{N}$ \\
\hline $0.3884(113)$ & $-0.009(3)$ & $0.303(13)$ & $-1.20(2)$ \\
$0.2654(81)$ & $-0.019(5)$ & $0.337(15)$ & $-1.33(3)$ \\
$0.1525(43)$ & $-0.025(6)$ & $0.350(14)$ & $-1.38(3)$ \\
$0.0784(25)$ & $-0.023(5)$ & $0.376(20)$ & $-1.40(3)$ \\
$0.0285(12)$ & $-0.022(9)$ & $0.437(84)$ & $-1.57(5)$ \\
\hline \hline
\end{tabular}

quark-mass results as they may be influenced by important unaccounted scattering-state contributions.

In Table IV, we present the charge radii, magnetic radii, and magnetic moments of the ground-state proton and neutron. These measurements are obtained from the electric and magnetic form factors as described above. We provide these results here for easy comparison with the excited-state results presented in this paper.

In Table V, we present the same results for the first negative-parity excitation. We see that this state has radii similar to the ground state but notably different magnetic moments. We find that at the heavier quark masses these magnetic moments agree well with constituent-quarkmodel predictions, as discussed below in Sec. IV E.

TABLE V. Radii and magnetic moments of the first negativeparity excitation of the proton and neutron. Radii are obtained from combinations of quark-sector dipole fits and magnetic moments are obtained from quark-sector ratios of $G_{M}\left(Q^{2}\right)$ to $G_{E}\left(Q^{2}\right)$.

\begin{tabular}{lccl}
\hline \hline$m_{\pi}^{2} / \mathrm{GeV}^{2}$ & $\left\langle r^{2}\right\rangle_{E}^{p_{1}^{*}} / \mathrm{fm}^{2}$ & $\left\langle r^{2}\right\rangle_{M}^{p_{1}^{*}} / \mu^{p_{1}^{*}} / \mathrm{fm}^{2}$ & $\mu^{p_{1}^{*}} / \mu_{N}$ \\
\hline $0.3884(113)$ & $0.340(29)$ & $0.21(3)$ & $1.53(7)$ \\
$0.2654(81)$ & $0.403(24)$ & $0.35(6)$ & $1.53(14)$ \\
$0.1525(43)$ & $0.421(29)$ & $0.30(5)$ & $1.66(8)$ \\
$0.0784(25)$ & $0.462(50)$ & $0.21(21)$ & $1.04(20)$ \\
$0.0285(12)$ & $0.470(54)$ & $0.63(24)$ & $2.07(28)$
\end{tabular}

\begin{tabular}{lrcc}
\hline \hline$m_{\pi}^{2} / \mathrm{GeV}^{2}$ & $\left\langle r^{2}\right\rangle_{E}^{n_{1}^{*}} / \mathrm{fm}^{2}$ & $\left\langle r^{2}\right\rangle_{M}^{n_{1}^{*}} / \mu^{n_{1}^{*}} / \mathrm{fm}^{2}$ & $\mu^{n_{1}^{*}} / \mu_{N}$ \\
\hline $0.3884(113)$ & $0.007(15)$ & $0.20(3)$ & $-0.93(4)$ \\
$0.2654(81)$ & $-0.016(12)$ & $0.34(5)$ & $-0.92(8)$ \\
$0.1525(43)$ & $0.014(18)$ & $0.32(8)$ & $-1.00(5)$ \\
$0.0784(25)$ & $-0.033(24)$ & $0.18(18)$ & $-0.61(12)$ \\
$0.0285(12)$ & $-0.021(36)$ & $0.62(25)$ & $-1.35(43)$ \\
\hline \hline
\end{tabular}

\section{C. $G_{E}$ for the second negative-parity excitation}

\section{Quark-flavor contributions}

We now proceed to examine the next negative-parity excitation observed in this study, $N_{2}^{*}$. In Fig. 12, we plot $G_{E}\left(Q^{2}\right)$ as a function of sink time for both quark flavors at $m_{\pi}=702 \mathrm{MeV}$ with the lowest-momentum kinematics. We see that the conventional extraction sits even further below the PEVA extraction than the first negative-parity excitation. While the PEVA fits start at time slice 24, the conventional fits are forced all the way out to time slice 28 and sit at only just above half of the values of the PEVA fits.

Moving on to the lighter pion masses, the discrepancy between the extracted form factors continues at $m_{\pi}=570$ and $411 \mathrm{MeV}$, with the conventional analysis giving consistently lower values than PEVA For example, Fig. 13 shows the plateaus at $m_{\pi}=570 \mathrm{MeV}$.

At the lightest two pion masses, the signal gets significantly noisier, and the difference between the two techniques gets harder to distinguish. Increased statistics are required in order to clearly identify the effects of opposite-parity contaminations of this state at these masses. However, in principle, the enhancement of relativistic components of the baryon spinors at light quark masses is expected to increase parity mixing in the conventional analysis.

In addition, in the tail of the two-point correlation function at the lightest mass, contributions from low-lying

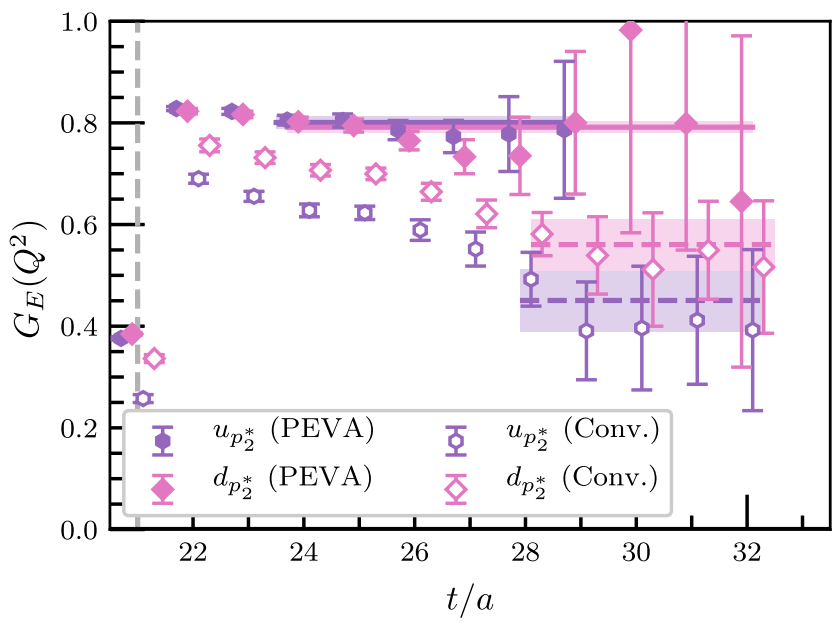

FIG. 12. Quark-flavor contributions to the electric form factor for the second negative-parity nucleon excitation at $m_{\pi}=$ $702 \mathrm{MeV}$ for the lowest-momentum kinematics, providing $Q^{2}=0.1425(41) \mathrm{GeV}^{2}$. We plot the conventional analysis with open markers and dashed fit lines and the new PEVA approach with filled markers and solid fit lines. Results for both the singly represented quark flavor $\left(d_{p_{2}^{*}}\right)$ and the doubly represented quark flavor $\left(u_{p_{2}^{*}}\right)$ are shown for single quarks of unit charge. Both PEVA fits are from time slice 24, whereas the conventional fits both start at time slice 28 . The values for both conventional fits are significantly lower than the corresponding PEVA fits. 


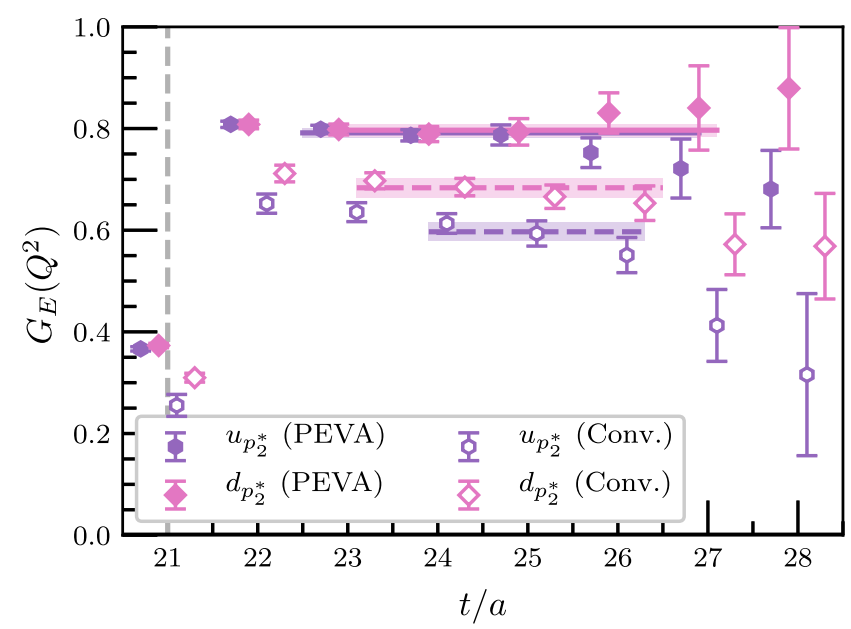

FIG. 13. Quark-flavor contributions to $G_{E}\left(Q^{2}\right)$ for the second negative-parity excitation at $m_{\pi}=570 \mathrm{MeV}$ for the lowestmomentum kinematics, providing $Q^{2}=0.1458(44) \mathrm{GeV}^{2}$. The conventions used in this plot are the same as in Fig. 12. The conventional fits have significantly lower values than the PEVA fits, and the plateau for the doubly represented quark flavor starts one time slice later than the corresponding PEVA plateau.

states are evident from a $\chi^{2}$ analysis of a single-state ansatz. This effect was also seen in Ref. [52], where it was found that so long as a single-state ansatz is satisfied in the fit region this contamination does not have a significant effect on the extracted mass. However, as shown in Ref. [9], contaminants that do not significantly perturb the extracted mass can still have a significant effect on the extracted form factors. As such, we must be cautious when interpreting results from this state at this mass. To gain a deeper insight into this state at this mass, multiparticle scattering operators will be necessary to properly isolate the low-lying scattering state.

Returning to the other ensembles, we find that the trends from the lowest-momentum kinematics continue for all other kinematics: for all masses for which the noise is sufficiently low, the conventional fits sit significantly lower than the PEVA fits.

Once again, these results clearly indicate that PEVA is critical to the correct extraction of the electric form factors of this nucleon excitation. The opposite-parity contaminations admitted by the conventional analysis lead to significant underestimation of the value of the electric form factor. Hence, we now focus our attention only on the PEVA results.

Plotting the acceptable plateaus as a function of $Q^{2}$ reveals that the contributions from the two quark flavors are once again very similar and agree well with a dipole ansatz. For example, Fig. 14 shows dipole fits to the two quark flavors at $m_{\pi}=411 \mathrm{MeV}$, with a rms charge radius of $0.679(38) \mathrm{fm}$ for the doubly represented quark flavor and 0.715 (31) fm for the singly represented quark flavor.

The charge radius of the doubly represented quark flavor of $0.679(38) \mathrm{fm}$ is similar to that seen in the ground state,

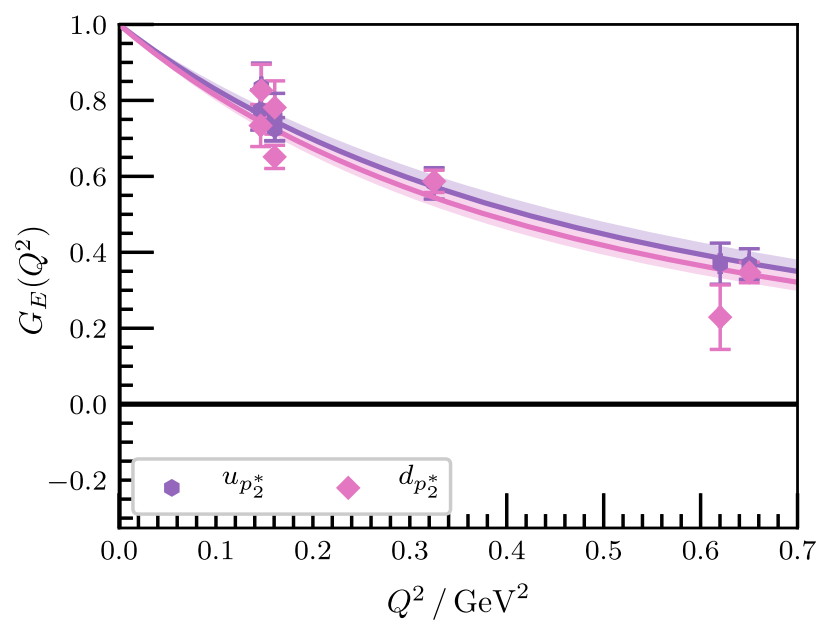

FIG. 14. Quark-flavor contributions to $G_{E}\left(Q^{2}\right)$ for the second negative-parity excitation at $m_{\pi}=411 \mathrm{MeV}$. The curves are dipole fits to the form factor, with the $y$ intercept fixed to unity. They correspond to rms charge radii of $0.679(38) \mathrm{fm}$ for the doubly represented quark $\left(u_{p}\right)$ and $0.715(31)$ fm for the singly represented quark $\left(d_{p}\right)$.

0.662(12) fm [9], again suggesting a role for meson-baryon contributions escaping the centripetal barrier encountered in a three-quark composition. This time the rms charge radius of the singly represented quark flavor of $0.715(31) \mathrm{fm}$ is larger that observed in the ground state, 0.633(12) fm. However, it does overlap with that of the first negativeparity excitation at the one-sigma level.

\section{Baryon electric form factors}

We once again take the linear combinations discussed in Sec. IVA to form the excited proton and neutron.

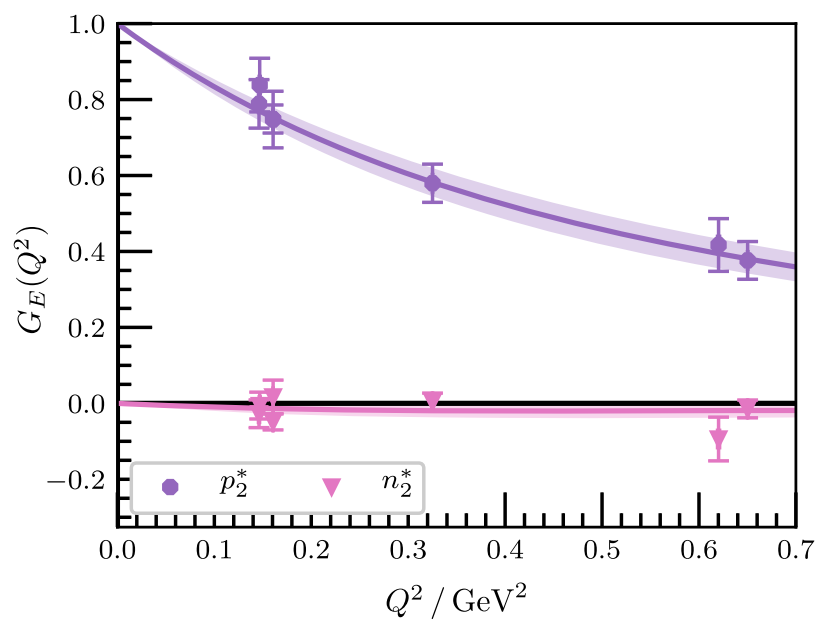

FIG. 15. $G_{E}\left(Q^{2}\right)$ for the second negative-parity excitation of the proton and neutron at $m_{\pi}=411 \mathrm{MeV}$. The curves correspond to combinations of the quark-sector dipole fits from Fig. 14, giving squared charge radii of $0.445(62) \mathrm{fm}^{2}$ for the excited proton and $0.033(30) \mathrm{fm}^{2}$ for the excited neutron. 


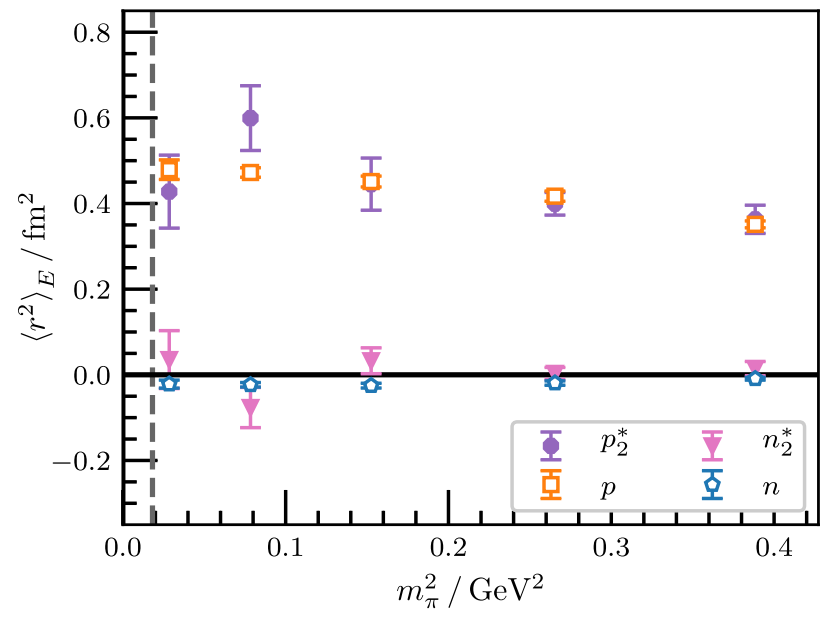

FIG. 16. Quark-mass dependence of charge radii from combinations of quark-sector dipole fits to $G_{E}\left(Q^{2}\right)$ for the second negative-parity excitation of the proton and neutron. The vertical dashed line corresponds to the physical pion mass, and the ground state is plotted with open points for comparison.

For example, in Fig. 15, we plot the electric form factors obtained from the quark-sector combinations at $m_{\pi}=$ $411 \mathrm{MeV}$. At all five masses, the electric form factor for the second negative-parity excitation of the neutron is approximately zero.

The pion-mass dependence of the charge radii is illustrated in Fig. 16. At the heaviest three pion masses, the linear combinations of the quark-sector dipole fits once again provide charge radii consistent with the ground-state nucleon at the same quark mass [9], pointing to a role for mesonbaryon $S$-wave Fock-space components, escaping the centripetal barrier encountered in a three-quark composition. We see that the pion-mass dependence of the proton radius is fairly smooth at these heaviest masses and has a clear trend to increasing charge radius at lower pion masses.

\section{D. $G_{M}$ for the second negative-parity excitation}

\section{Quark-flavor contributions}

We now advance to the magnetic form factor of this state. In Fig. 17, we plot the heaviest pion mass of $m_{\pi}=$ $702 \mathrm{MeV}$ and the lowest-momentum kinematics. While the plateau time regions for the PEVA and conventional analysis are consistent, the values of those plateaus are very different and, in fact, change ordering between the two extractions. We see a similar effect at $m_{\pi}=296,411$, and $570 \mathrm{MeV}$, with similar inversions of the magnetic form factors between the two analyses. We see similar patterns for the other kinematics, with significantly different plateau values between the two analyses when the statistical noise is low enough to distinguish them.

Once again the PEVA technique is crucial to extracting the correct form factors. Hence, we focus only on the PEVA results. Inspecting the $Q^{2}$ dependence of these form factors,

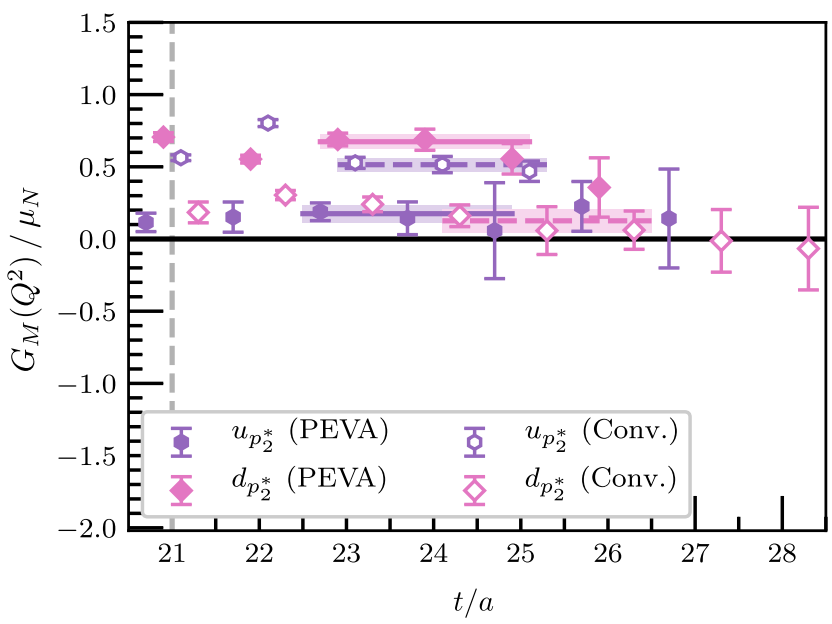

FIG. 17. Quark-flavor contributions to $G_{M}\left(Q^{2}\right)$ for the second negative-parity excitation of the nucleon at $m_{\pi}=702 \mathrm{MeV}$ for the lowest-momentum kinematics, providing $Q^{2}=0.1425(41) \mathrm{GeV}^{2}$. The conventions used in this plot are the same as in Fig. 12. The plateaus for the PEVA technique both start at time slice 23 . The plateaus for the conventional analysis start at time slice 23 for $u_{p_{2}^{*}}$ and time slice 24 for $d_{p_{2}^{*}}$. The difference in the plateau values between the two analyses is enough to change the ordering of the two quark flavors.

we find that the contributions from both quark flavors agree well with a dipole ansatz. For example, Fig. 18 shows the form factors at $m_{\pi}=411 \mathrm{MeV}$. Here we have held the $y$ scale fixed to match previous plots, for ease of comparison. The most notable feature of these results is their small magnitude compared to both the ground state and the excitation considered in Sec. IV B.

\section{Baryon magnetic form factors}

By taking linear combinations based on the multiplicity and charge of each quark flavor, as described in Sec. IV A,

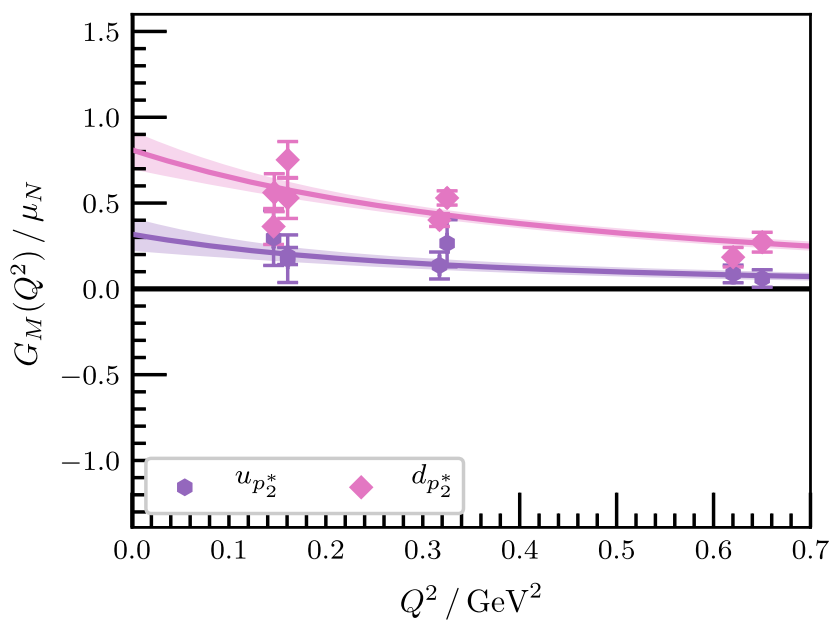

FIG. 18. Quark-flavor contributions to $G_{M}\left(Q^{2}\right)$ for the second negative-parity excitation at $m_{\pi}=411 \mathrm{MeV}$. The curves are dipole fits to the individual quark sectors. 


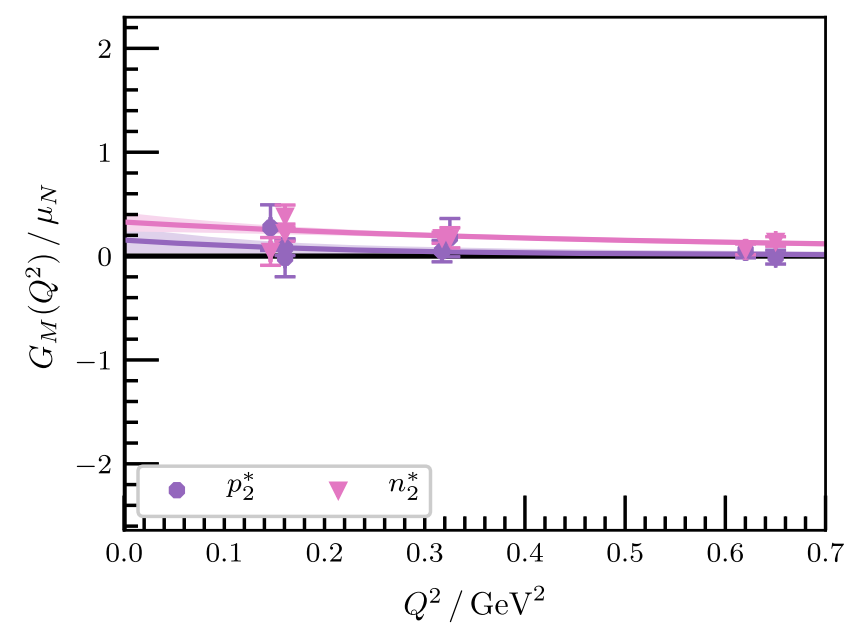

FIG. 19. $G_{M}\left(Q^{2}\right)$ for the excited proton and neutron at $m_{\pi}=411 \mathrm{MeV}$. The curves correspond to combinations of the quark-sector dipole fits from Fig. 18.

we can obtain the magnetic form factors for the excited proton and neutron. Figure 19 shows these combinations for $m_{\pi}=411 \mathrm{MeV}$. The magnetic charge radii obtained by combining the quark-sector dipoles are consistent with the proton charge radii from $G_{E}\left(Q^{2}\right)$, although they often have very large errors due to the very small values of the magnetic form factors amplifying the effects of statistical fluctuations.

\section{Baryon magnetic moments}

Returning to the individual quark-sector results with unit charge, and noting that the $Q^{2}$ dependence for $G_{E}\left(Q^{2}\right)$ and $G_{M}\left(Q^{2}\right)$ is similar, we once again take the ratio

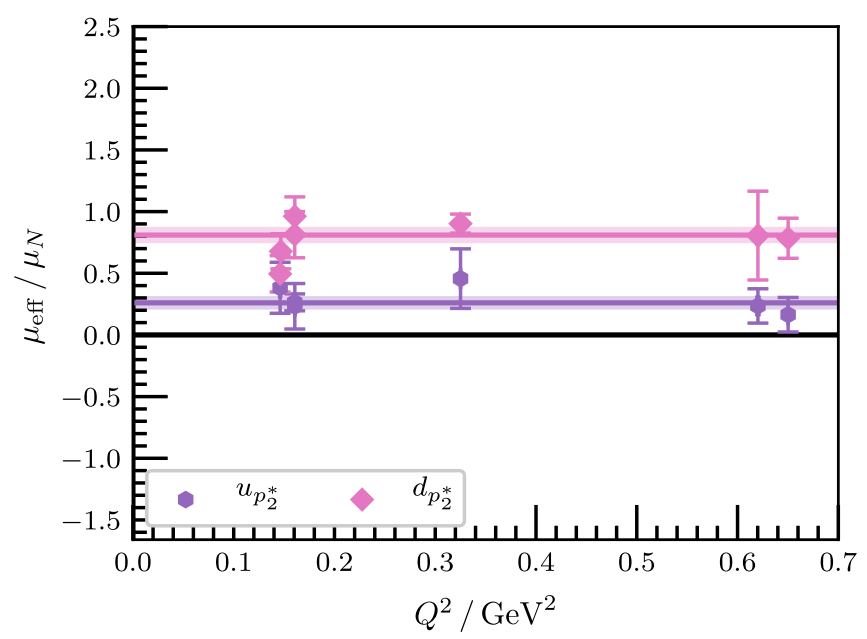

FIG. 20. $\mu_{\text {eff }}$ for individual quarks of unit charge in the second negative-parity excitation at $m_{\pi}=411 \mathrm{MeV}$. The shaded bands are constant fits to the effective magnetic moment, corresponding to magnetic moment contributions of $0.260(55) \mu_{N}$ for the doubly represented quark and $0.810(67) \mu_{N}$ for the singly represented quark.

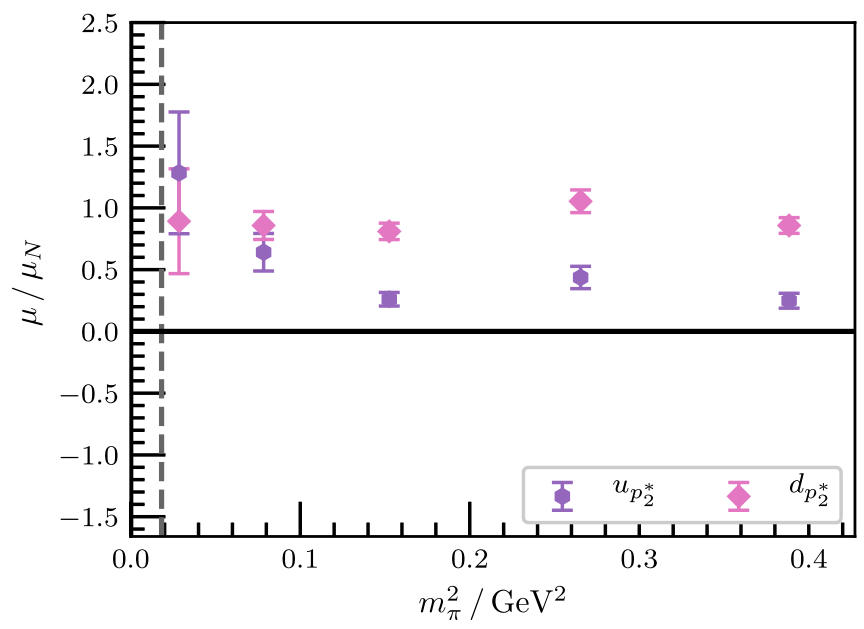

FIG. 21. Quark-mass dependence of contributions from individual unit-charge quarks to the magnetic moment of the second negative-parity excitation of the nucleon. The vertical dashed line corresponds to the physical pion mass.

$\mu_{\text {eff }}\left(Q^{2}\right) \equiv G_{M}\left(Q^{2}\right) / G_{E}\left(Q^{2}\right)$. We find that this ratio is approximately flat for all five pion masses. For example, Fig. 20 shows the $Q^{2}$ dependence of the ratio at $m_{\pi}=$ $411 \mathrm{MeV}$. We can extract the contributions to the magnetic moment from both quark flavors from constant fits to this ratio.

Figure 21 shows the pion-mass dependence of these extracted magnetic moment contributions. It is remarkable that both quark flavors contribute with the same sign.

By taking the linear combinations discussed above, we can combine these individual quark-flavor results to get the predicted magnetic moments for the second negative-parity excitations of the proton and neutron. In Fig. 22 we plot the

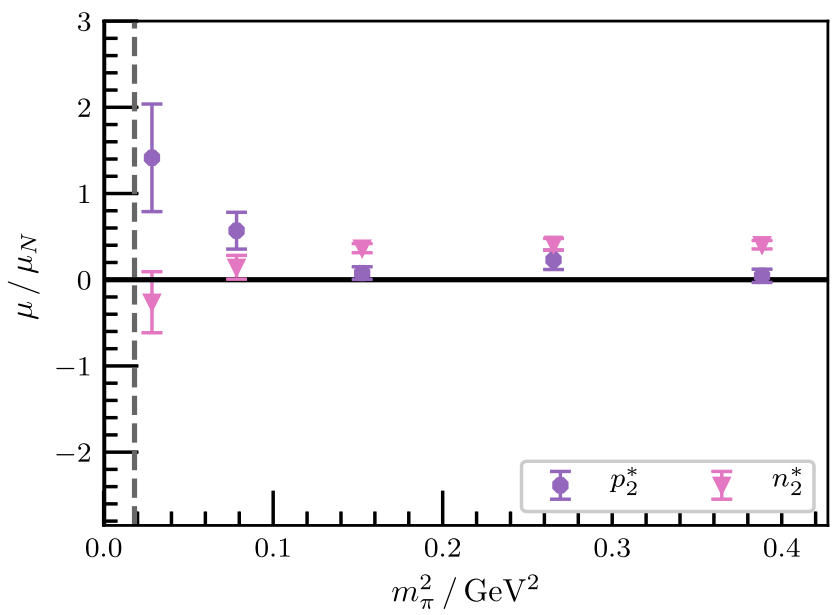

FIG. 22. Quark-mass dependence of the magnetic moment of the second negative-parity excitations of the proton and neutron. The dashed line corresponds to the physical pion mass. There is a clear change at the lightest two pion masses, which signals a significant shift in the structure of the states. 
TABLE VI. Radii and magnetic moments of the second negative-parity excitation of the proton and neutron. Radii are obtained from combinations of quark-sector dipole fits and magnetic moments are obtained from quark-sector ratios of $G_{M}\left(Q^{2}\right)$ to $G_{E}\left(Q^{2}\right)$. At the lightest two pion masses, the form factor data were insufficient to properly constrain a dipole fit so we do not report magnetic radii at these masses.

\begin{tabular}{lccc}
\hline \hline$m_{\pi}^{2} / \mathrm{GeV}^{2}$ & $\left\langle r^{2}\right\rangle_{E}^{p_{2}^{*}} / \mathrm{fm}^{2}$ & $\left\langle r^{2}\right\rangle_{M}^{p_{2}^{*}} / \mu^{p_{2}^{*}} / \mathrm{fm}^{2}$ & $\mu^{p_{2}^{*}} / \mu_{N}$ \\
\hline $0.3884(113)$ & $0.363(35)$ & $0.56(213)$ & $0.05(8)$ \\
$0.2654(81)$ & $0.399(29)$ & $0.55(70)$ & $0.23(11)$ \\
$0.1525(43)$ & $0.445(62)$ & $1.09(70)$ & $0.08(7)$ \\
$0.0784(25)$ & $0.599(77)$ & $\ldots$ & $0.57(21)$ \\
$0.0285(12)$ & $0.428(86)$ & $\ldots$ & $1.41(62)$ \\
\hline \hline$m_{\pi}^{2} / \mathrm{GeV}^{2}$ & $\left\langle r^{2}\right\rangle_{E}^{n_{2}^{*}} / \mathrm{fm}^{2}$ & $\left\langle r^{2}\right\rangle_{M}^{n_{2}^{*}} / \mu^{n_{2}^{*}} / \mathrm{fm}^{2}$ & $\mu^{n_{2}^{*}} / \mu_{N}$ \\
\hline $0.3884(113)$ & $0.014(17)$ & $0.30(18)$ & $0.41(5)$ \\
$0.2654(81)$ & $0.003(14)$ & $0.65(30)$ & $0.41(7)$ \\
$0.1525(43)$ & $0.033(30)$ & $0.41(34)$ & $0.37(5)$ \\
$0.0784(25)$ & $-0.076(47)$ & $\ldots$ & $0.14(14)$ \\
$0.0285(12)$ & $0.036(68)$ & $\ldots$ & $-0.26(35)$ \\
\hline \hline
\end{tabular}

dependence of these combinations on the squared pion mass. For the heaviest three pion masses, the effective magnetic moments show little pion-mass dependence and have tight error bars. The magnetic moment of the proton excitation sits very close to zero, and the magnetic moment of the neutron excitation has a small but nonzero positive value. For the lightest two masses, the ordering of the two states flips, with the proton excitation taking on a more significant magnetic moment and the neutron excitation dropping to be consistent with or below zero.

In summary, the PEVA technique is crucial for extracting both the electric and magnetic form factors of the second negative-parity excitations of the proton and neutron. In Table VI, we present the charge radii, magnetic radii, and magnetic moments of the second negative-parity excitation of the proton and neutron. We see that this state has similar radii to the ground state but notably different magnetic moments. We find that at the heavier quark masses these magnetic moments agree well with constituent-quarkmodel predictions, as discussed below.

\section{E. Model comparison}

In Sec. I, we introduced the two low-lying negativeparity excitations of the proton and neutron observed on the lattice by the CSSM and the HSC Collaborations. We wish to examine the extent to which these states, formed in relativistic quantum field theory, have properties that are captured in simple quark models. As these states have good overlap with local three-quark operators, we anticipate these states may resemble the quark-model states postulated to describe these resonances $[1,6,7]$.

Already we have seen some deviations from quarkmodel expectations for electric charge radii. There, larger radii for the $N^{*}$ states were anticipated due to centripetal repulsion in the radial Schrödinger equation for three-quark states with orbital angular momentum $\ell=1$. However, similar charge radii point to five-quark meson-baryon components in the $N^{*}$ structure where the antiquark provides negative parity and all quarks can sit in relative $s$ waves.

Here, we focus on the magnetic moments of these two negative-parity states, as calculated in Secs. IV B and IV D. It will be interesting to learn if there is a nontrivial role for meson-baryon Fock-space components here as well.

Our focus on the three heavier quark masses considered is beneficial in comparing with quark models as it is this regime where constituent quark phenomenology is expected to be manifest $[44,55]$.

We consider two constituent-quark-model $(\mathrm{QM})$ predictions of the magnetic moments from Refs. $[1,6]$ and two chiral constituent-quark-model $(\chi \mathrm{QM})$ calculations which take the quark-model calculations and include effects from the pion cloud [6,7], thus incorporating meson-baryon Fock-space components.

In Fig. 23, we compare our magnetic moments extracted at $m_{\pi}=702 \mathrm{MeV}$ with these quark-model predictions, which are calculated at the physical point. We can see that, qualitatively, the results for the first negative-parity lattice excitation match up with the quark model $N^{*}(1535)$, and the second negative-parity lattice excitation with the quark model $N^{*}(1650)$. In fact, despite being at significantly different pion masses, the results are quantitatively very similar, with the lattice results sitting within the scatter of the model predictions for all states save the second negative-parity nucleon excitation, which sits slightly below all of the model predictions.

For comparison, we also plot lattice results produced using the conventional variational analysis. For these results, $\mu_{\text {eff }}\left(Q^{2}\right)$ varies significantly for different kinematics, so rather than taking a constant fit across kinematics, we present only the result from the lowest-momentum $\left[p=(0,0,0), p^{\prime}=(1,0,0)\right]$ kinematics, which we expect to have the smallest opposite-parity contaminations. We see that the conventional results are significantly different to the PEVA results. In particular, the conventional extraction of the second negative-parity excitation is completely different to both the PEVA result and the quark-model results. This once again demonstrates how critical the PEVA technique is to obtaining correct results.

This trend continues for $m_{\pi}=570 \mathrm{MeV}$, and $m_{\pi}=$ $411 \mathrm{MeV}$, the latter shown in Fig. 24. Since the pion-mass dependence of the magnetic moments between these three masses is quite small, the quantitative agreement remains good.

For completeness, we also present a comparison for the second lightest mass. Figure 25 shows that the lattice results depart from the model predictions at $m_{\pi}=$ $296 \mathrm{MeV}$. Again, an analysis of possible scattering-state 


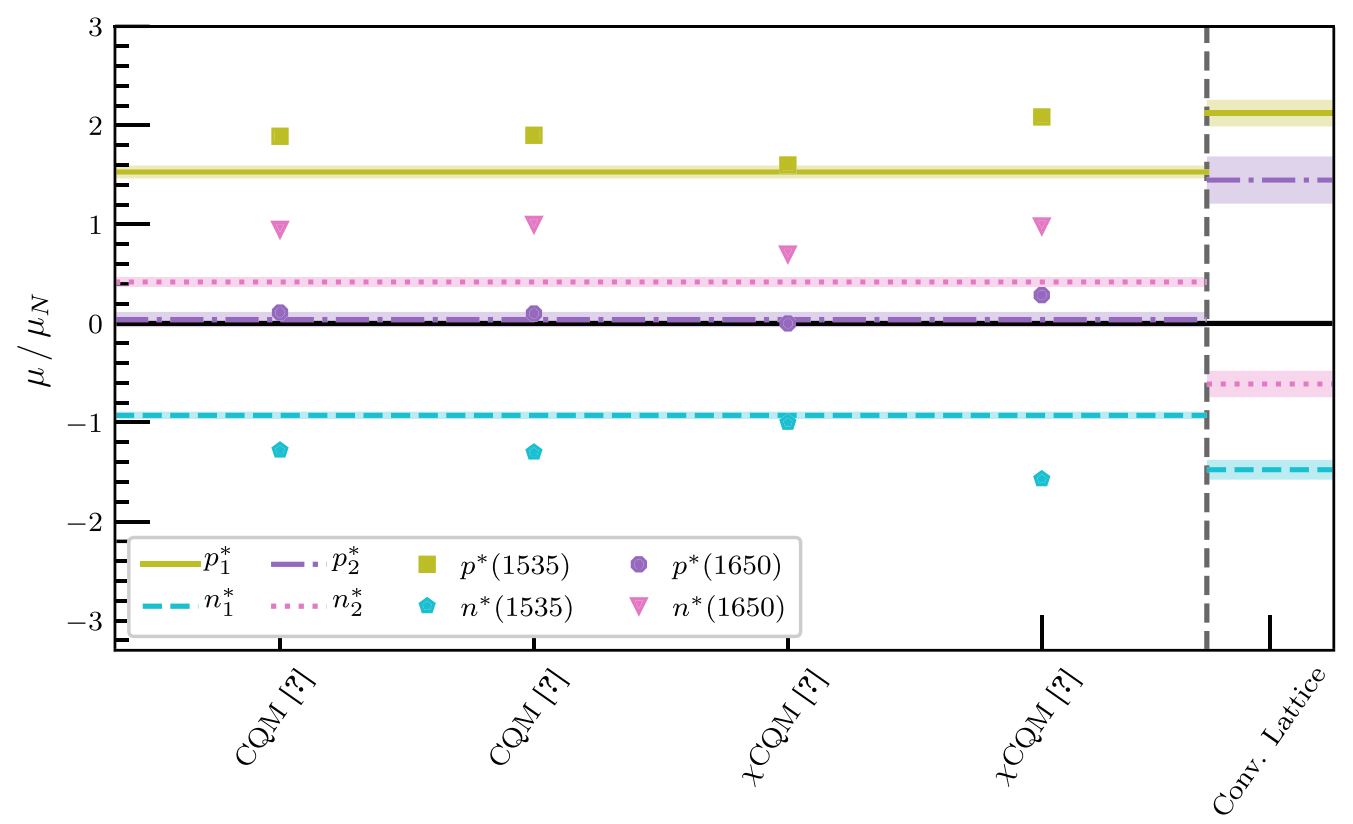

FIG. 23. Comparison between lattice calculations of the magnetic moments of two negative-parity nucleon excitations at $m_{\pi}=$ $702 \mathrm{MeV}$ and quark-model predictions for the $N^{*}(1535)$ and $N^{*}(1650)$ resonances. The shaded bands on the left-hand side of the plot indicate the magnetic moments calculated via the PEVA technique, and symbols denote the quark-model predictions. Lattice calculations of the magnetic moments using a conventional variational analysis are plotted to the right of the vertical dashed line.

contributions to the correlation functions is required to disentangle interesting meson-cloud effects from scattering-state contaminations as discussed in Sec. III D. Figure 25 also shows that there is still a significant disagreement between the conventional and PEVA results at $m_{\pi}=296 \mathrm{MeV}$. It remains clear that the PEVA technique is playing an important role in addressing opposite-parity contaminations.

The results presented in this section provide new insight into the structure of the negative-parity nucleon excitations observed in lattice quantum field theory. At the heavier quark masses considered, the two negative-parity

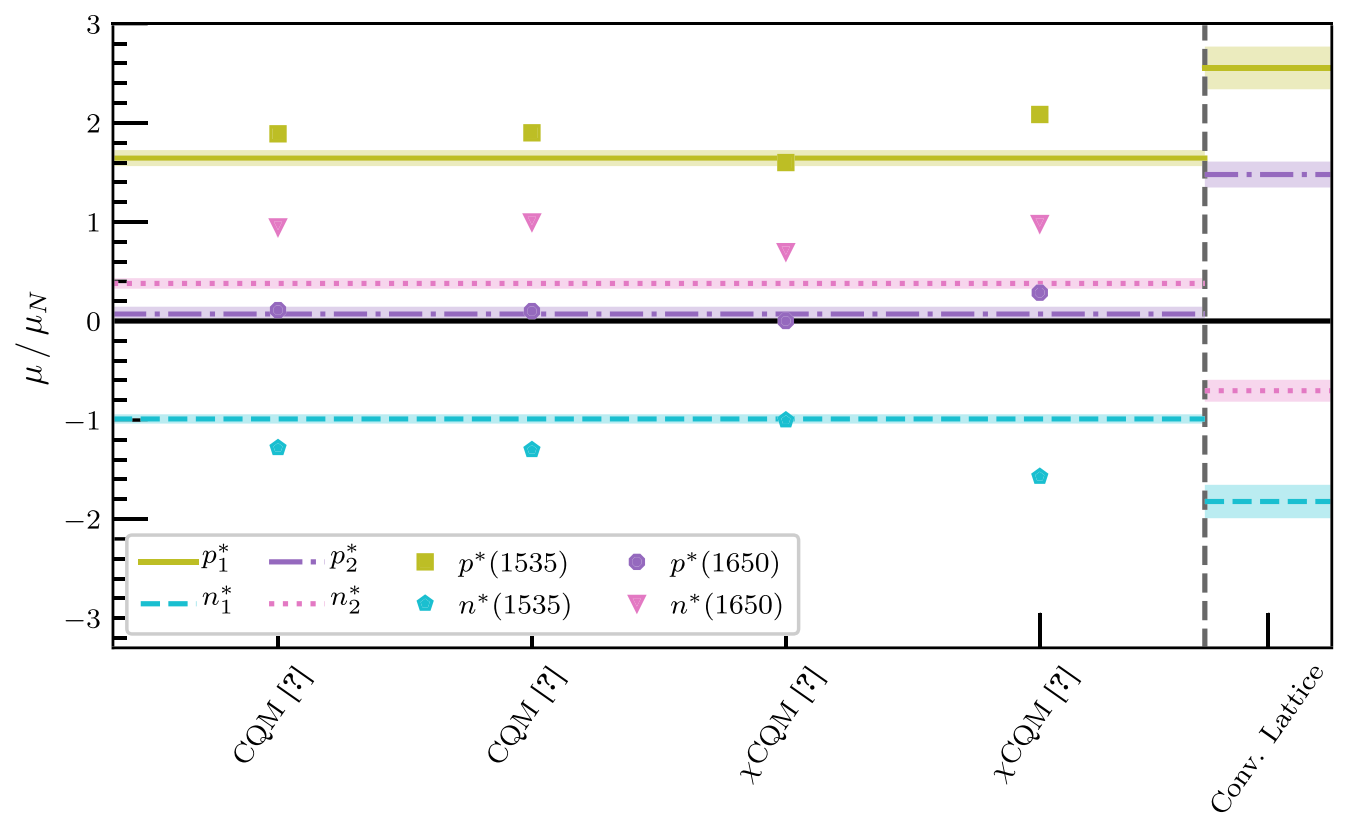

FIG. 24. Comparison between magnetic moments from lattice calculations at $m_{\pi}=411 \mathrm{MeV}$ and quark-model predictions for the $N^{*}(1535)$ and $N^{*}(1650)$. The shaded bands indicate the PEVA calculations on the left and the conventional analysis on the right. The markers show the quark-model predictions. 


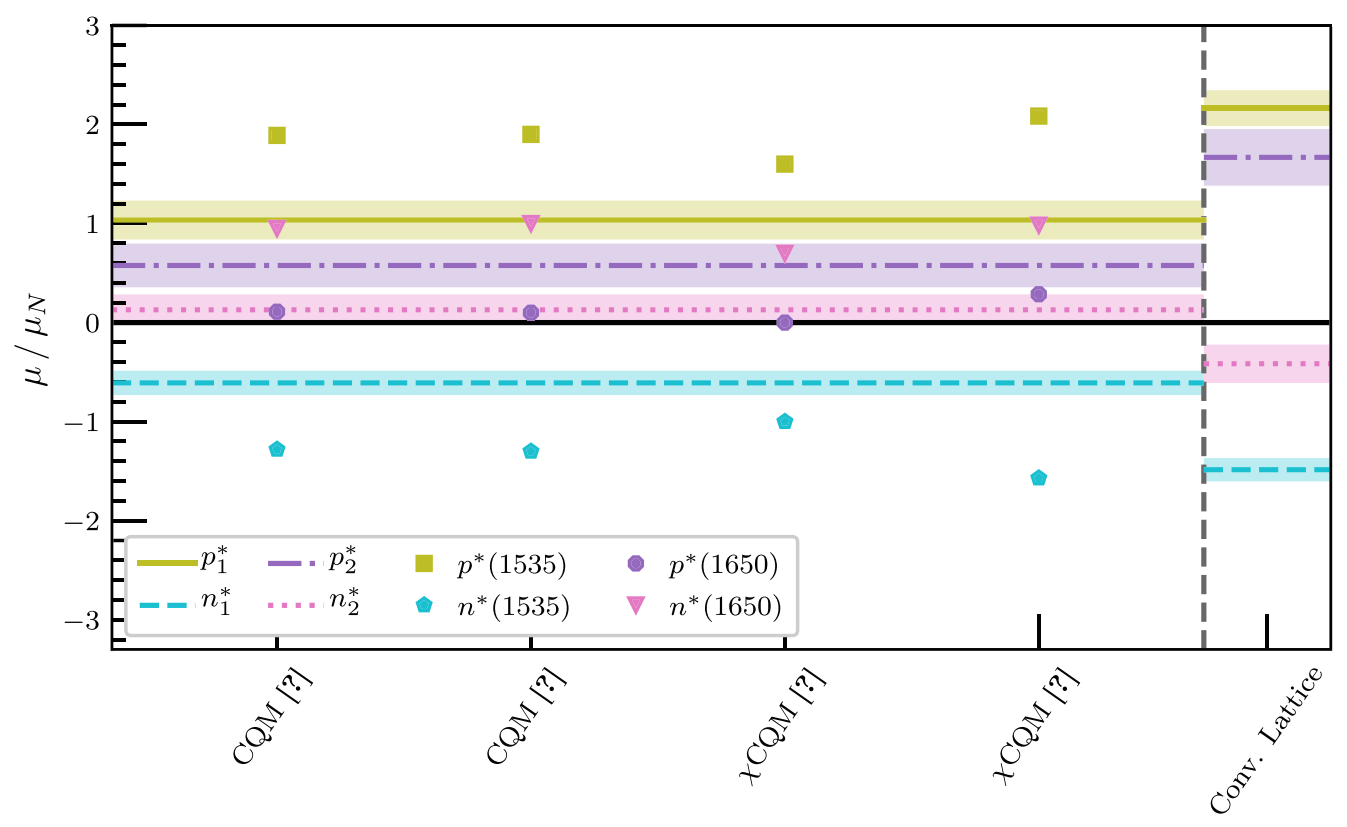

FIG. 25. Comparison between magnetic moments from lattice calculations at $m_{\pi}=296 \mathrm{MeV}$ and quark-model predictions for the $N^{*}(1535)$ and $N^{*}(1650)$. The shaded bands indicate the PEVA calculations on the left and the conventional analysis on the right. The markers show the quark-model predictions.

excitations agree well with quark-model descriptions for the $N^{*}(1535)$ and $N^{*}(1650)$. Coupled with the charge radii from Sec. IVA, which indicate the importance of mesonnucleon components in the wave function, and the observed single-particle dispersion relations seen in Ref. [41], the results indicate that these states are similar in structure to the ground-state nucleon which can also be modeled as a three-quark state dressed by a meson cloud.

\section{POSITIVE-PARITY EXCITATION}

We now move to the positive-parity sector, studying the first localized positive-parity excitation of the nucleon observed on the lattice. This state sits at an effective mass of approximately $2 \mathrm{GeV}$ for all five pion masses, well above $1.43(2) \mathrm{GeV}$, the mass of the Roper resonance observed in nature [56]. This has long been a puzzle for the particle physics community, but recent HEFT results indicate that the Roper resonance is dynamically generated from meson-baryon scattering states [18], and hence the lattice spectrum in this energy region has poor overlap with local three-quark operators. This means that the lattice state studied here is likely associated with the $N^{*}(1710)$, $N^{*}(1880)$, and/or $N^{*}(2100)$ resonances.

\section{A. Electric form factor}

\section{Quark-flavor contributions}

We plot the dependence of $G_{E}\left(Q^{2}\right)$ on the Euclidean sink time at $m_{\pi}=702 \mathrm{MeV}$ in Figs. 26 and 27. The form factor values extracted from the PEVA and conventional analyses for each sink time look very similar, and this is reflected in the fits. The conventional and PEVA extractions both have clear plateaus over the same range of sink times, and these plateaus have consistent values.

This trend continues for lighter pion masses: the PEVA and conventional analyses have the same fit ranges and consistent fit values. This is also true for all kinematics for which we are able to find acceptable plateaus. This suggests

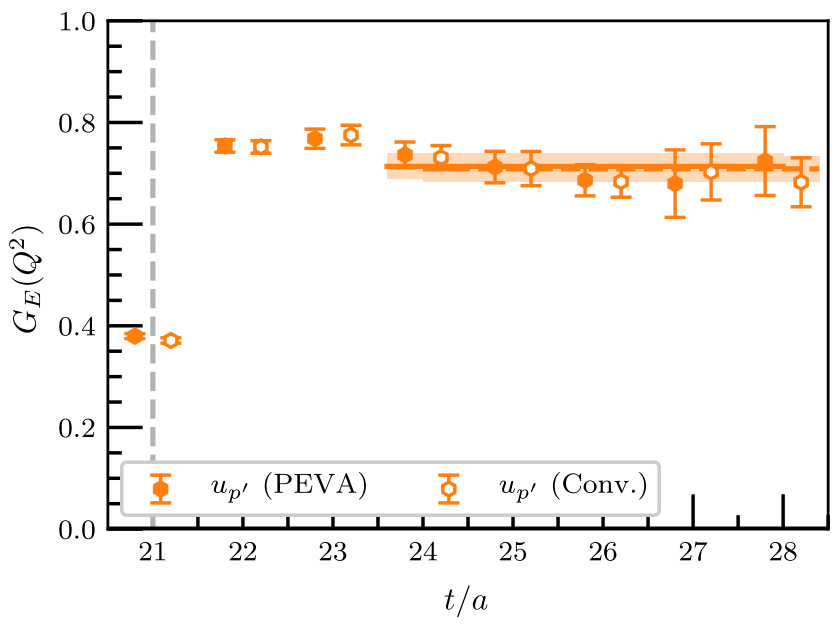

FIG. 26. Contributions to $G_{E}\left(Q^{2}\right)$ from the doubly represented quark flavor for the first positive-parity nucleon excitation at $m_{\pi}=702 \mathrm{MeV}$ with the lowest-momentum kinematics, providing $Q^{2}=0.1425(41) \mathrm{GeV}^{2}$. We plot the conventional analysis with open markers and dashed fit lines and the PEVA technique with filled markers and solid fit lines. Both fits are from time slices 24-28. 


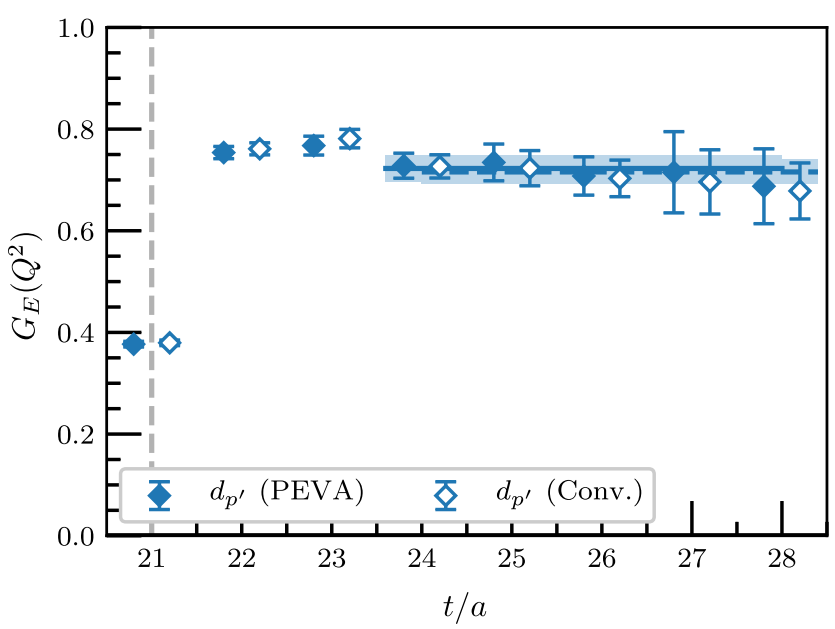

FIG. 27. Contributions to $G_{E}\left(Q^{2}\right)$ from the singly represented quark flavor for the first positive-parity nucleon excitation. The pion mass, kinematics and plotting convention are the same as in Fig. 26 above. Both fits are from time slices 24-28.

there are no significant effects from opposite-parity contaminations on $G_{E}\left(Q^{2}\right)$ for this state, at least at this level of statistics.

Focusing on the PEVA results, in Fig. 28, we plot the $Q^{2}$ dependence of the electric form factor for the two valence quark flavors at $m_{\pi}=411 \mathrm{MeV}$. We see that the two quark flavors make very similar contributions to the electric form factor and agree well with a dipole fit corresponding to charge radii of $0.88(4) \mathrm{fm}$ for the doubly represented quark flavor and $0.89(5) \mathrm{fm}$ for the singly represented quark flavor. This is significantly larger than the ground-state nucleon. Similar behavior is seen for the other four masses.

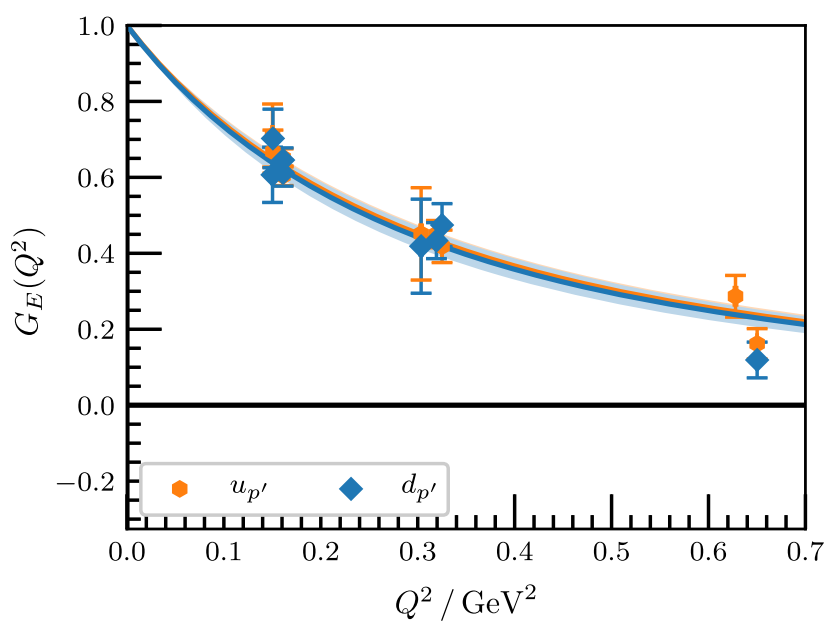

FIG. 28. Quark-flavor contributions to $G_{E}\left(Q^{2}\right)$ for the first positive-parity excitation at $m_{\pi}=411 \mathrm{MeV}$. The curves are dipole fits to the form factor, with lines indicating the central values. The fits correspond to rms charge radii of $0.871(36) \mathrm{fm}$ for the doubly represented quark flavor $\left(u_{p^{\prime}}\right)$ and $0.885(44) \mathrm{fm}$ for the singly represented quark flavor $\left(d_{p^{\prime}}\right)$.

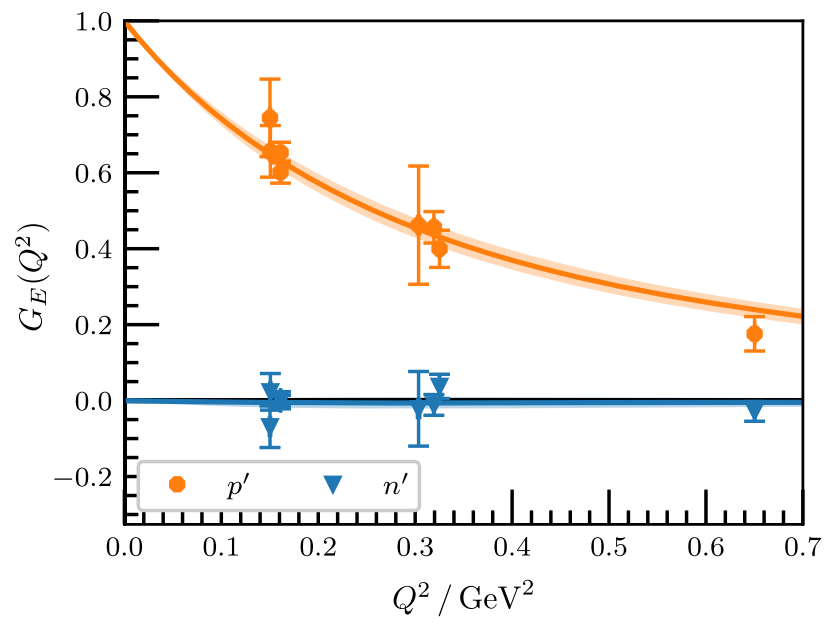

FIG. 29. $G_{E}\left(Q^{2}\right)$ for the first positive-parity excitations of the proton and neutron at $m_{\pi}=411 \mathrm{MeV}$. The curves correspond to combinations of the quark-sector dipole fits from Fig. 28, giving a squared charge radius of $0.75(7) \mathrm{fm}^{2}$ for the proton and $0.016(39) \mathrm{fm}^{2}$ for the neutron.

\section{Baryon electric form factors}

As above, we take linear combinations of the individual quark-flavor contributions, including the charges of the quark flavors and their multiplicity, to get the electric form factors for the first positive-parity excitations of the proton and neutron. In Fig. 29, we plot these combinations at $m_{\pi}=411 \mathrm{MeV}$. At this and the other four masses, we find that the electric form factor for the neutron excitation is approximately zero.

In Fig. 30, we plot the pion-mass dependence of charge radii extracted from combinations of the quark-sector dipole fits to the electric form factor. For the heaviest three masses, squared charge radii for the proton range from $0.67(7)$ to $0.75(7) \mathrm{fm}^{2}$, increasing with decreasing pion

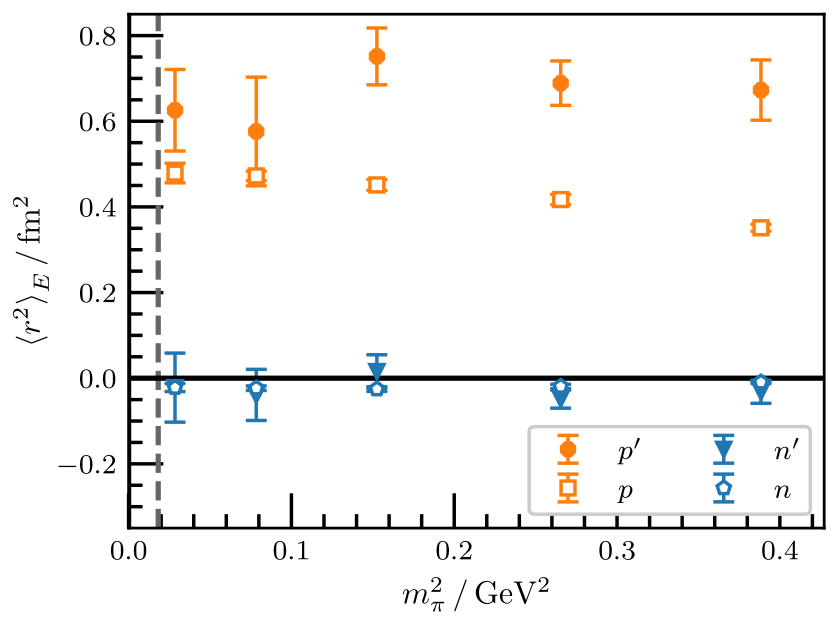

FIG. 30. Quark-mass dependence of charge radii from combinations of quark-sector dipole fits to $G_{E}\left(Q^{2}\right)$ for the first positiveparity excitation of the proton. 


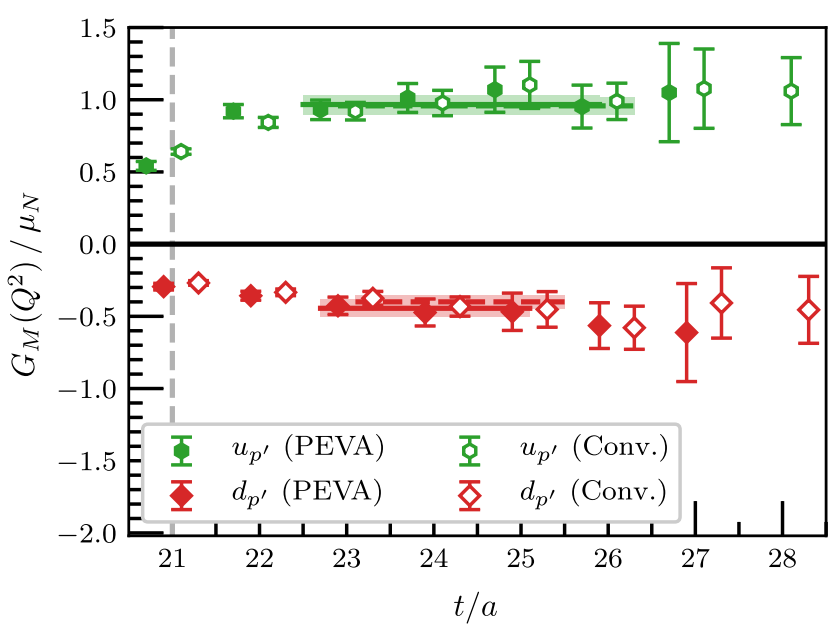

FIG. 31. Quark-flavor contributions to the magnetic form factor for the first positive-parity excitation of the nucleon at $m_{\pi}=$ $702 \mathrm{MeV}$ for the lowest-momentum kinematics, providing $Q^{2}=0.1425(41) \mathrm{GeV}^{2}$. Results are for single quarks of unit charge. All four fits start from time slice 23 and have consistent values.

mass. These radii are all significantly larger than the charge radius of the ground-state proton at the corresponding mass. Thus the second positive-parity excitation is significantly larger than the ground-state proton at these pion masses, in line with earlier observations that this positiveparity excitation has a wave function consistent with a three-quark radial excitation with one node [33,34]. At the lightest two masses, the statistical errors become large. However the trend for this positive-parity excitation to be larger than the ground state is manifest.

\section{B. Magnetic form factor}

\section{Quark-flavor contributions}

Having investigated the electric form factor for this state, we now consider the magnetic form factor. In Fig. 31 we plot the Euclidean sink-time dependence of the extracted form factors at $m_{\pi}=702 \mathrm{MeV}$, with the lowest-momentum kinematics. We see that the form factors and plateaus for both analyses are very similar, and there is no evidence for opposite-parity contamination of this state. We see similar results for the other masses and kinematics, with no clear differences between the conventional and PEVA plateaus. For example, Fig. 32 shows this behavior at $m_{\pi}=411 \mathrm{MeV}$ with the same lowest-momentum kinematics. This suggests that, like $G_{E}\left(Q^{2}\right), G_{M}\left(Q^{2}\right)$ for the first positive-parity excitation is not affected by opposite-parity excitations, at least at this level of statistics.

Focusing on the PEVA results, we plot the $Q^{2}$ dependence of the plateau fits for the two valence quark flavors at $m_{\pi}=411 \mathrm{MeV}$ in Fig. 33. We see that both quark flavors agree well with a dipole ansatz. This is also true for the two

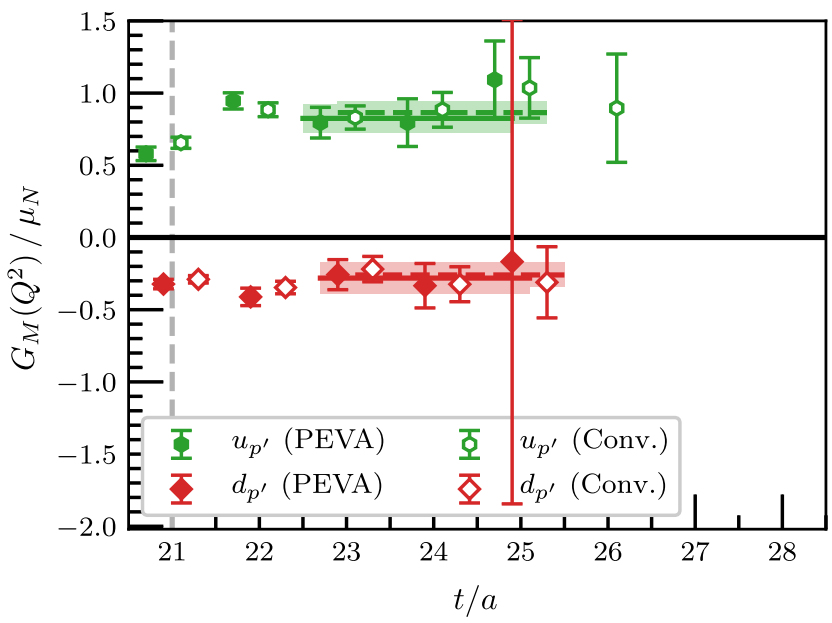

FIG. 32. Quark-flavor contributions to $G_{M}\left(Q^{2}\right)$ for the first positive-parity excitation of the nucleon at $m_{\pi}=411 \mathrm{MeV}$ for the lowest-momentum kinematics, providing $Q^{2}=0.146 \mathrm{GeV}^{2}$.

heavier pion masses, and the two lighter masses are also consistent, though they are too noisy to significantly constrain the fit.

\section{Baryon magnetic form factors}

Combinations of the quark-flavor contributions are taken to form the proton and neutron excitations. In Fig. 34, we plot these combinations at $m_{\pi}=411 \mathrm{MeV}$. By combining the dipole fits to the quark-sector results in the same way, we obtain magnetic radii that are consistent with the corresponding excited proton charge radius. This can be seen in Fig. 35, in which we plot the pion-mass dependence of the squared magnetic radii obtained from the linear combinations of the dipole fits to the form factors. These plots show fairly consistent results for the heavier three

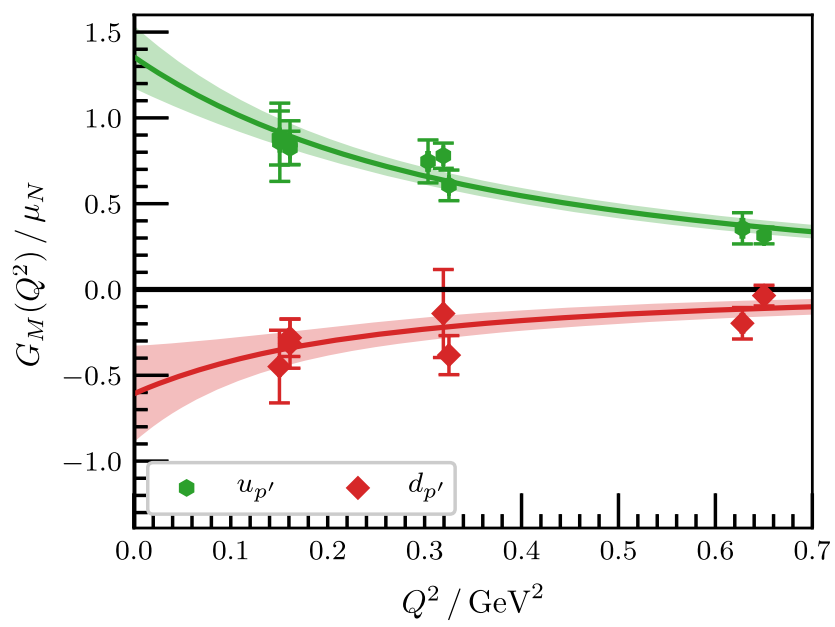

FIG. 33. Quark-flavor contributions to $G_{M}\left(Q^{2}\right)$ for the first positive-parity excitation at $m_{\pi}=411 \mathrm{MeV}$. The curves are dipole fits to the form factor, corresponding to squared magnetic radii of $0.67(14) \mathrm{fm}^{2}$ for $u_{p^{\prime}}$ and $0.97(59) \mathrm{fm}^{2}$ for $d_{p^{\prime}}$. 


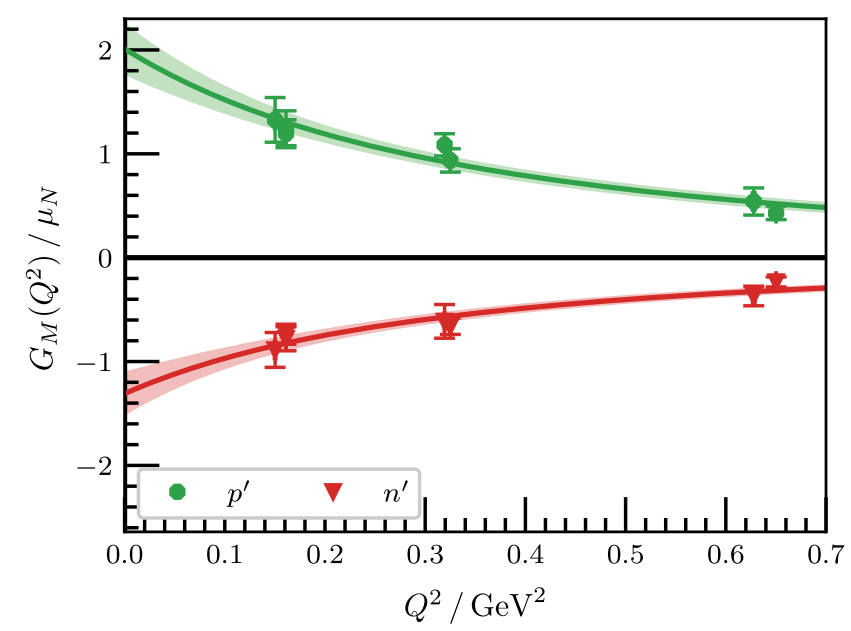

FIG. 34. $G_{M}\left(Q^{2}\right)$ for the first positive-parity excitations of the proton and neutron at $m_{\pi}=411 \mathrm{MeV}$. The curves correspond to combinations of the quark-sector dipole fits from Fig. 33, giving a squared magnetic radius of $0.70(13) \mathrm{fm}^{2}$ for the proton excitation and $0.77(21) \mathrm{fm}^{2}$ for the neutron excitation.

masses, with some pion-mass dependence. We omit results for the lightest two masses because the form factor data are insufficient to constrain dipole fits at these masses.

\section{Baryon magnetic moments}

Returning to the individual quark-sector results and noting that once again the electric and magnetic form factors have a similar $Q^{2}$ dependence, we take the ratio $\mu_{\text {eff }}\left(Q^{2}\right) \equiv G_{M}\left(Q^{2}\right) / G_{E}\left(Q^{2}\right)$. In Fig. 36, we plot this ratio as a function of $Q^{2}$ for $m_{\pi}=411 \mathrm{MeV}$. We find that the ratio is once again very flat in $Q^{2}$, supporting our hypothesis that the form factors have the same $Q^{2}$ scaling

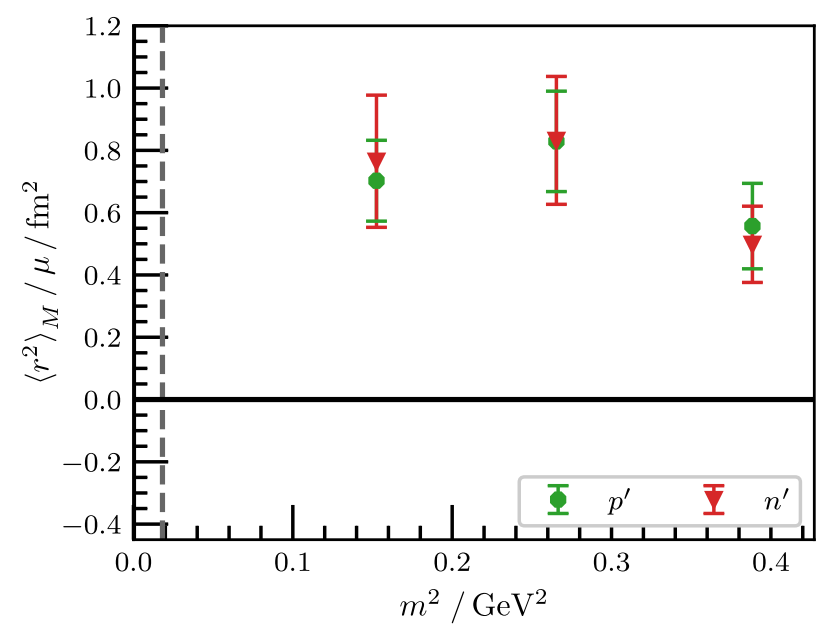

FIG. 35. Quark-mass dependence of squared magnetic radii of the first positive-parity excitation of the proton and neutron from quark-sector dipole fits to $G_{M}\left(Q^{2}\right)$. At the lightest two pion masses, the form factor data were insufficient to properly constrain a dipole fit so we do not report magnetic radii at these masses.

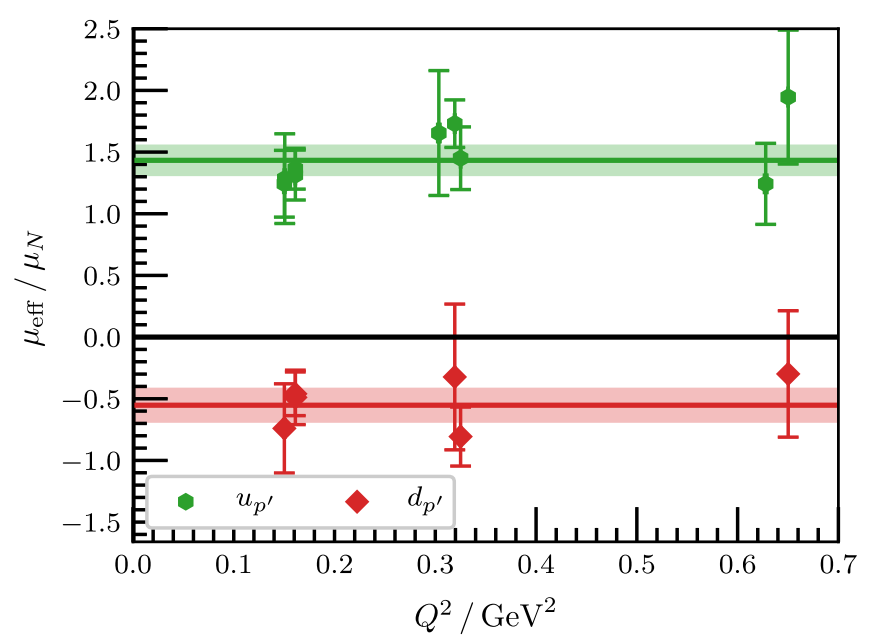

FIG. 36. $\mu_{\text {eff }}$ for individual quarks of unit charge in the first positive-parity excitation at $m_{\pi}=411 \mathrm{MeV}$. The shaded bands are constant fits to the effective magnetic moments which provide magnetic moment contributions of $1.43(13) \mu_{N}$ for the doubly represented quark and $-0.55(14) \mu_{N}$ for the singly represented quark.

in this region and the validity of $\mu_{\text {eff }}$ as an estimate of the magnetic moment.

In Fig. 37, we plot the pion-mass dependence of $\mu_{\text {eff }}$ for individual quarks of unit charge. We can once again take combinations of the individual quark-flavor contributions to get the excited proton and neutron magnetic moments. In Fig. 38, we plot the pion-mass dependence of these combinations.

We see that the excited-state magnetic moments agree well with the ground-state magnetic moments, particularly at the heaviest quark masses, where the agreement is impressive. These results are in accord with a simple $2 S$ constituent-quark-model state.

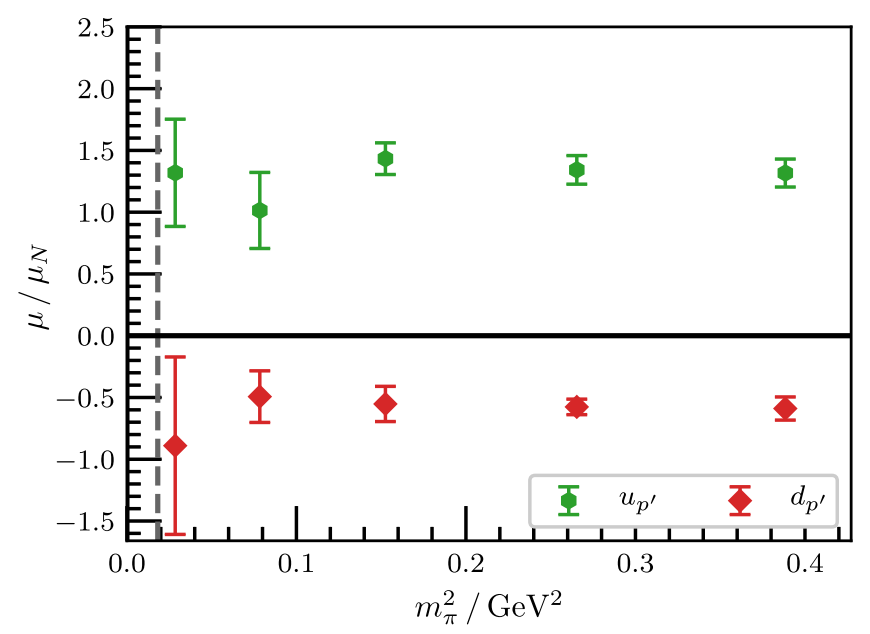

FIG. 37. Quark-mass dependence of contributions from individual unit-charge quarks to the magnetic moment of the first positive-parity excitation of the nucleon. The vertical dashed line corresponds to the physical pion mass. 


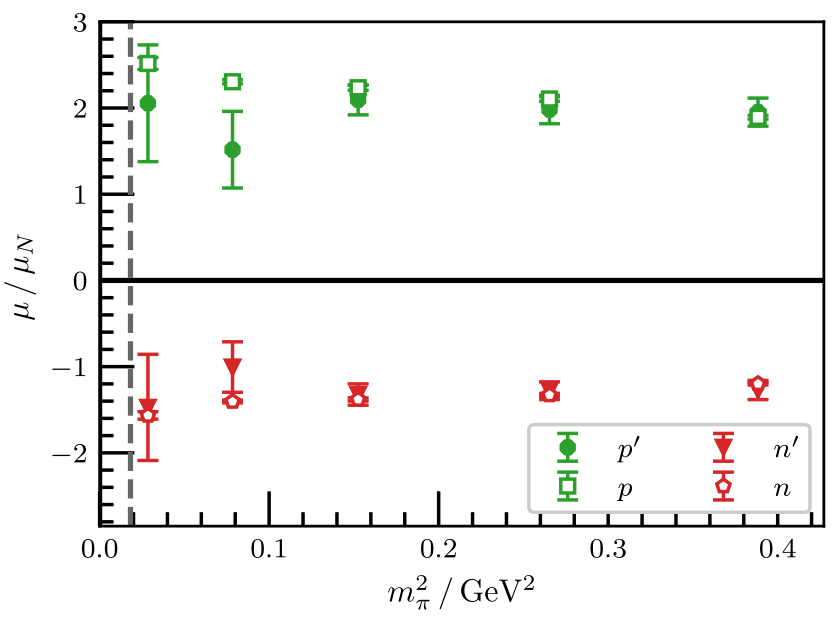

FIG. 38. Quark-mass dependence of the magnetic moments of the first positive-parity excitations of the proton and neutron. The vertical dashed line corresponds to the physical pion mass, and the ground-state magnetic moments have been plotted with open markers.

In summary, we have shown that the first positive-parity excitation of the nucleon has no obvious opposite-parity contaminations. However, variational analysis techniques in general have provided good access to this state at several pion masses. This has allowed us to ascertain for the first time that these states have a larger radius than the groundstate nucleon but have very similar magnetic moments. This is consistent with these states being dominated by a radial $2 S$ excitation of the ground-state nucleon as seen in Refs. [33,34]. Table VII collects our charge radii, magnetic radii, and magnetic moments for this positive-parity excitation of the proton and neutron.

TABLE VII. Radii and magnetic moments of the positive-parity excitation of the proton and neutron. Radii are obtained from combinations of quark-sector dipole fits and magnetic moments are obtained from quark-sector ratios of $G_{M}\left(Q^{2}\right)$ to $G_{E}\left(Q^{2}\right)$. At the lightest two pion masses, the form factor data were insufficient to properly constrain a dipole fit so we do not report magnetic radii at these masses.

\begin{tabular}{lccc}
\hline \hline$m_{\pi}^{2} / \mathrm{GeV}^{2}$ & $\left\langle r^{2}\right\rangle_{E}^{p^{\prime}} / \mathrm{fm}^{2}$ & $\left\langle r^{2}\right\rangle_{M}^{p^{\prime}} / \mu^{p^{\prime}} / \mathrm{fm}^{2}$ & $\mu^{p^{\prime}} / \mu_{N}$ \\
\hline $0.3884(113)$ & $0.673(73)$ & $0.56(14)$ & $1.95(17)$ \\
$0.2654(81)$ & $0.689(56)$ & $0.83(16)$ & $1.98(17)$ \\
$0.1525(43)$ & $0.751(69)$ & $0.70(13)$ & $2.09(18)$ \\
$0.0784(25)$ & $0.576(128)$ & $\cdots$ & $1.52(44)$ \\
$0.0285(12)$ & $0.626(97)$ & $\cdots$ & $2.06(68)$ \\
\hline \hline$m_{\pi}^{2} / \mathrm{GeV}^{2}$ & $\left\langle r^{2}\right\rangle_{E}^{n^{\prime}} / \mathrm{fm}^{2}$ & $\left\langle r^{2}\right\rangle_{M}^{n^{\prime}} / \mu^{n^{\prime}} / \mathrm{fm}^{2}$ & $\mu^{n^{\prime}} / \mu_{N}$ \\
\hline $0.3884(113)$ & $-0.032(27)$ & $0.50(12)$ & $-1.27(11)$ \\
$0.2654(81)$ & $-0.049(21)$ & $0.83(21)$ & $-1.28(10)$ \\
$0.1525(43)$ & $0.016(39)$ & $0.77(21)$ & $-1.32(12)$ \\
$0.0784(25)$ & $-0.039(60)$ & $\cdots$ & $-1.00(29)$ \\
$0.0285(12)$ & $-0.022(81)$ & $\cdots$ & $-1.47(62)$ \\
\hline \hline
\end{tabular}

\section{CONCLUSION}

In this paper we presented the first calculations of the elastic form factors of lattice nucleon excitations from the first principles of QCD. We have considered a variety of momentum frames to access a range of $Q^{2}$ values, with some approaching zero. We have presented the first implementation of the PEVA technique for matrix elements, which is vital to isolating exited baryons with nonzero momentum.

Substantial differences between the form factors calculated in a conventional variational analysis and those calculated with the PEVA approach show the PEVA technique to be critical to understanding the structure of these excited states.

We find the size of the two lowest-lying negative parity excitations to be similar to the ground-state nucleons. This is a remarkable result in the context of a simple constituent quark model, where the repulsive centripetal term of the radial Schrödinger equation proportional to $\ell(\ell+1)$ is expected to force the quarks to larger radii for $\ell=1$ states. The lattice-QCD results point to a role for meson-nucleon Fock-space components in the $N^{*}$ states. As the antiquark in the meson provides the negative parity, all quarks can reside in relative $s$ waves. In this way the centripetal barrier is avoided and the negative-parity states can have a size similar to the ground-state nucleon.

The positive-parity excitation observed in this study is very high in energy, approaching $2 \mathrm{GeV}$, and exhibits challenging statistical fluctuations. The extractions of the form factors herein are attained through the cancellation of statistical fluctuations enabled by the combination of an $\mathcal{O}(a)$-improved conserved vector current and an appropriately selected correlator ratio preserving the lattice Ward identity. This state has a charge radius approximately $30 \%$ larger than the ground state and magnetic moments which match the ground state. Both of these observations are in accord with earlier observations that this positive-parity excitation has a wave function consistent with a three-quark $2 S$ radial excitation $[33,34]$.

At the heaviest three pion masses considered, the first observed negative-parity excitations have magnetic moments consistent with quark-model descriptions for the $N^{*}(1535)$. Similarly, the second negative-parity excitations have magnetic moments in accord with quark-model descriptions of the $N^{*}(1650)$. At these quark masses, the results indicate these states are similar in structure to the ground-state nucleon which can also be modeled as a threequark state dressed by a meson cloud.

At the lightest two pion masses, we observe a rearrangement in the structure of the second negative-parity excitation. This is evident in both a significant shift in the magnetic moments of the excited proton and neutron and significant curvature in the pion-mass dependence of the electric form factor. A description of this state as a molecular bound state of $K \Sigma$ dressed by $K \Lambda, \eta N$ and 
$\pi N$ is an intriguing possibility, analogous to the description of the odd-parity $\Lambda(1405)$ excitation as a molecular bound state of $\bar{K} N$ dressed by $\pi \Sigma[8,57]$. The proximity of the noninteracting $K \Sigma$ to the effective energy of the observed lattice state is suggestive.

While the current analysis is able to raise such a possibility, it is not able to affirm it. As a first step, nonlocal momentum-projected two-particle interpolating fields must be introduced to provide access to the $K \Sigma, K \Lambda$, $\eta N$ and $\pi N$ scattering states. To date, only the energy of the $S$-wave $\pi N$ scattering state has been investigated [10,58]. This will be a challenging endeavor, owing to the computational cost of estimating the loop propagators that are necessary to compute nonlocal momentum-projected meson-baryon contracted interpolating fields. Moreover, very high statistics will be required to precisely evaluate the correlation functions for quark masses near the physical regime.

However, such studies will allow for the lattice determination of the form factors of the multiparticle-dominated scattering states at light quark masses and will provide the input required to make a robust connection between these states and the infinite-volume resonances of nature [31,32].

Looking forward, it is interesting to note that HEFT calculations describe the negative-parity energy spectrum with reference to one bare basis state [10]. This investigation indicates the presence of two quark-model-like states raising the possibility of introducing a second bare basis state into the formalism. It will be interesting to explore such an extension of HEFT as the two quarkmodel-like basis states mix through couplings to intermediate meson-baryon basis states. Such dynamics may be relevant to a detailed quantitative understanding of the negative-parity nucleon spectrum.

\section{ACKNOWLEDGMENTS}

This research was undertaken with the assistance of resources from the Phoenix HPC service at the University of Adelaide, the National Computational Infrastructure (NCI), which is supported by the Australian Government, and by resources provided by the Pawsey Supercomputing Centre with funding from the Australian Government and the Government of Western Australia. These resources were provided through the National Computational Merit Allocation Scheme and the University of Adelaide partner share. This research is supported by the Australian Research Council through Grants No. DP140103067, No. DP150103164, No. LE160100051, No. LE190100021 and No. DP190102215.
[1] W.-T. Chiang, S. N. Yang, M. Vanderhaeghen, and D. Drechsel, Magnetic dipole moment of the $S_{11}(1535)$ from the $\gamma p \rightarrow \gamma \eta p$ reaction, Nucl. Phys. A723, 205 (2003).

[2] M. S. Mahbub, W. Kamleh, D. B. Leinweber, P. J. Moran, and A.G. Williams, Structure and flow of the nucleon eigenstates in lattice QCD, Phys. Rev. D 87, 094506 (2013).

[3] M. S. Mahbub, W. Kamleh, D. B. Leinweber, P. J. Moran, and A. G. Williams, Low-lying odd-parity states of the nucleon in lattice QCD, Phys. Rev. D 87, 011501(R) (2013).

[4] R. G. Edwards, N. Mathur, D. G. Richards, and S. J. Wallace (Hadron Spectrum Collaboration), Flavor structure of the excited baryon spectra from lattice QCD, Phys. Rev. D 87, 054506 (2013).

[5] R. G. Edwards, J. J. Dudek, D. G. Richards, and S. J. Wallace, Excited state baryon spectroscopy from lattice QCD, Phys. Rev. D 84, 074508 (2011).

[6] J. Liu, J. He, and Y.B. Dong, Magnetic moments of negative-parity low-lying nucleon resonances in quark models, Phys. Rev. D 71, 094004 (2005).

[7] N. Sharma, A. Martinez Torres, K. P. Khemchandani, and H. Dahiya, Magnetic moments of the low-lying $\frac{1}{2}^{-1}$ octet baryon resonances, Eur. Phys. J. A 49, 11 (2013).

[8] J. M. M. Hall, W. Kamleh, D. B. Leinweber, B. J. Menadue, B. J. Owen, A. W. Thomas, and R. D. Young, Lattice QCD
Evidence that the $\Lambda(1405)$ Resonance is an AntikaonNucleon Molecule, Phys. Rev. Lett. 114, 132002 (2015).

[9] F. M. Stokes, W. Kamleh, and D. B. Leinweber, Oppositeparity contaminations in lattice nucleon form factors, Phys. Rev. D 99, 074506 (2019).

[10] Z.-W. Liu, W. Kamleh, D. B. Leinweber, F. M. Stokes, A. W. Thomas, and J.-J. Wu, Hamiltonian Effective Field Theory Study of the $N^{*}(1535)$ Resonance in Lattice QCD, Phys. Rev. Lett. 116, 082004 (2016).

[11] M. Luscher, Two-particle states on a torus and their relation to the scattering matrix, Nucl. Phys. B354, 531 (1991).

[12] J. M. M. Hall, A. C. P. Hsu, D. B. Leinweber, A. W. Thomas, and R. D. Young, Finite-volume matrix Hamiltonian model for a $\Delta \rightarrow N \pi$ system,Phys. Rev. D 87, 094510 (2013).

[13] Y. Li, J.-J. Wu, C. D. Abell, D. B. Leinweber, and A. W. Thomas, Partial-wave mixing in Hamiltonian effective field theory, Phys. Rev. D 101, 114501 (2020).

[14] M. S. Mahbub, W. Kamleh, D. B. Leinweber, P. J. Moran, and A. G. Williams (CSSM Lattice Collaboration), Roper resonance in $2+1$ flavor QCD, Phys. Lett. B 707, 389 (2012).

[15] C. Alexandrou, T. Leontiou, C. N. Papanicolas, and E. Stiliaris, Novel analysis method for excited states in lattice QCD: The nucleon case, Phys. Rev. D 91, 014506 (2015).

[16] A. L. Kiratidis, W. Kamleh, D. B. Leinweber, and B. J. Owen, Lattice baryon spectroscopy with multiparticle interpolators, Phys. Rev. D 91, 094509 (2015). 
[17] A. L. Kiratidis, W. Kamleh, D. B. Leinweber, Z.-W. Liu, F. M. Stokes, and A. W. Thomas, Search for low-lying lattice QCD eigenstates in the Roper regime, Phys. Rev. D 95, 074507 (2017).

[18] Z.-W. Liu, W. Kamleh, D. B. Leinweber, F. M. Stokes, A. W. Thomas, and J.-J. Wu, Hamiltonian effective field theory study of the $N^{*}(1440)$ resonance in lattice QCD, Phys. Rev. D 95, 034034 (2017).

[19] C. B. Lang, L. Leskovec, M. Padmanath, and S. Prelovsek, Pion-nucleon scattering in the Roper channel from lattice QCD, Phys. Rev. D 95, 014510 (2017).

[20] J. J. Dudek and R. G. Edwards, Hybrid baryons in QCD, Phys. Rev. D 85, 054016 (2012).

[21] D. J. Wilson, R. A. Briceño, J. J. Dudek, R. G. Edwards, and C. E. Thomas, Coupled $\pi \pi, K \bar{K}$ scattering in $P$ and the $\rho$ resonance from lattice QCD, Phys. Rev. D 92, 094502 (2015).

[22] Z.-W. Liu, J. M. Hall, D. B. Leinweber, A. W. Thomas, and J.-J. $\mathrm{Wu}$, Structure of the $\Lambda(1405)$ from Hamiltonian effective field theory, Phys. Rev. D 95, 014506 (2017).

[23] J.-j. Wu, D. B. Leinweber, Z.-w. Liu, and A. W. Thomas, Structure of the Roper resonance from lattice QCD constraints, Phys. Rev. D 97, 094509 (2018).

[24] C. W. Andersen, J. Bulava, B. Hrz, and C. Morningstar, Elastic $I=3 / 2 p$-wave nucleon-pion scattering amplitude and the $\Delta(1232)$ resonance from $N_{f}=2+1$ lattice QCD, Phys. Rev. D 97, 014506 (2018).

[25] C. W. Andersen, J. Bulava, B. Hrz, and C. Morningstar, $I=$ $3 / 2 N \pi$ scattering and the $\Delta(1232)$ resonance on $N_{f}=$ $2+1$ CLS ensembles using the stochastic LapH method, Proc. Sci. LATTICE2019 (2019) 039 [arXiv:1911.10021].

[26] R. A. Briceño, M. T. Hansen, and A. Walker-Loud, Multichannel $1 \rightarrow 2$ transition amplitudes in a finite volume, Phys. Rev. D 91, 034501 (2015).

[27] R. A. Briceño and M. T. Hansen, Multichannel $0 \rightarrow 2$ and $1 \rightarrow 2$ transition amplitudes for arbitrary spin particles in a finite volume, Phys. Rev. D 92, 074509 (2015).

[28] R. A. Briceño, J. J. Dudek, R. G. Edwards, C. J. Shultz, C. E. Thomas, and D. J. Wilson, Resonant $\pi^{+} \gamma \rightarrow \pi^{+} \pi^{0}$ Amplitude from Quantum Chromodynamics, Phys. Rev. Lett. 115, 242001 (2015).

[29] R. A. Briceño, J. J. Dudek, R. G. Edwards, C. J. Shultz, C. E. Thomas, and D. J. Wilson, $\pi \pi \rightarrow \pi \gamma^{*}$ amplitude and the resonant $\rho \rightarrow \pi \gamma^{*}$ transition from lattice QCD, Phys. Rev. D 93, 114508 (2016).

[30] C. Alexandrou, L. Leskovec, S. Meinel, J. Negele, S. Paul, M. Petschlies, A. Pochinsky, G. Rendon, and S. Syritsyn, $\pi \gamma \rightarrow \pi \pi$ transition and the $\rho$ radiative decay width from lattice QCD, Phys. Rev. D 98, 074502 (2018).

[31] R. A. Briceño and M. T. Hansen, Relativistic, model-independent, multichannel $2 \rightarrow 2$ transition amplitudes in a finite volume, Phys. Rev. D 94, 013008 (2016).

[32] A. Baroni, R. A. Briceño, M. T. Hansen, and F. G. OrtegaGama, Form factors of two-hadron states from a covariant finite-volume formalism, Phys. Rev. D 100, 034511 (2019).

[33] D. S. Roberts, W. Kamleh, and D. B. Leinweber, Wave function of the Roper from lattice QCD, Phys. Lett. B 725, 164 (2013).

[34] D. S. Roberts, W. Kamleh, and D. B. Leinweber, Nucleon excited state wave functions from lattice QCD, Phys. Rev. D 89, 074501 (2014).
[35] D. Leinweber, W. Kamleh, A. Kiratidis, Z.-W. Liu, S. Mahbub, D. Roberts, F. Stokes, A. W. Thomas, and J. $\mathrm{Wu}, N^{*}$ spectroscopy from lattice QCD: The Roper explained, J. Phys. Soc. Jpn. Conf. Proc. 10, 010011 (2016).

[36] J. J. Dudek, R. Edwards, and C. E. Thomas, Exotic and excited-state radiative transitions in charmonium from lattice QCD, Phys. Rev. D 79, 094504 (2009).

[37] B. J. Owen, W. Kamleh, D. B. Leinweber, M. S. Mahbub, and B. J. Menadue, Transition of $\rho \rightarrow \pi \gamma$ in lattice QCD, Phys. Rev. D 92, 034513 (2015).

[38] C. J. Shultz, J. J. Dudek, and R. G. Edwards, Excited meson radiative transitions from lattice QCD using variationally optimized operators, Phys. Rev. D 91, 114501 (2015).

[39] C. E. Thomas, R. G. Edwards, and J. J. Dudek, Helicity operators for mesons in flight on the lattice, Phys. Rev. D 85, 014507 (2012).

[40] M. Padmanath, S. Collins, D. Mohler, S. Piemonte, S. Prelovsek, A. Schäfer, and S. Weishaeupl, Identifying spin and parity of charmonia in flight with lattice QCD, Phys. Rev. D 99, 014513 (2019).

[41] F. M. Stokes, W. Kamleh, D. B. Leinweber, M. S. Mahbub, B. J. Menadue, and B. J. Owen, Parity-expanded variational analysis for nonzero momentum, Phys. Rev. D 92, 114506 (2015).

[42] G. Martinelli, C. T. Sachrajda, and A. Vladikas, A study of "improvement" in lattice QCD, Nucl. Phys. B358, 212 (1991).

[43] S. Boinepalli, D. B. Leinweber, A. G. Williams, J. M. Zanotti, and J. B. Zhang, Precision electromagnetic structure of octet baryons in the chiral regime, Phys. Rev. D 74, 093005 (2006).

[44] D. B. Leinweber, R. M. Woloshyn, and T. Draper, Electromagnetic structure of octet baryons, Phys. Rev. D 43, 1659 (1991).

[45] S. Aoki, K. Ishikawa, N. Ishizuka, T. Izubuchi, D. Kadoh, K. Kanaya, Y. Kuramashi, Y. Namekawa, M. Okawa, Y. Taniguchi, A. Ukawa, N. Ukita, and T. Yoshie (PACS-CS Collaboration), $2+1$ flavor lattice QCD toward the physical point, Phys. Rev. D 79, 034503 (2009).

[46] M. G. Beckett, B. Joo, C. M. Maynard, D. Pleiter, O. Tatebe, and T. Yoshie, Building the International Lattice Data Grid, Comput. Phys. Commun. 182, 1208 (2011).

[47] S. Gusken, A study of smearing techniques for hadron correlation functions, Nucl. Phys. B, Proc. Suppl. 17, 361 (1990).

[48] C. Morningstar and M. J. Peardon, Analytic smearing of $S U$ (3) link variables in lattice QCD, Phys. Rev. D 69, 054501 (2004).

[49] C. W. Bernard, T. Draper, G. Hockney, and A. Soni, in Lattice Gauge Theory: A Challenge in Large-Scale Computing (Wuppertal, Germany, 1985), pp. 199-207.

[50] Z.-W. Liu, J. M. M. Hall, W. Kamleh, D. B. Leinweber, F. M. Stokes, A. W. Thomas, and J.-J. Wu, Study of low-lying baryons with Hamiltonian effective field theory, Proc. Sci. INPC2016 (2017) 288 [arXiv:1701.08582].

[51] O. Bar, Chiral perturbation theory and nucleon-pion-state contaminations in lattice QCD, Int. J. Mod. Phys. A 32, 1730011 (2017).

[52] M. S. Mahbub, W. Kamleh, D. B. Leinweber, and A. G. Williams, Searching for low-lying multi-particle thresholds 
in lattice spectroscopy, Ann. Phys. (Amsterdam) 342, 270 (2014).

[53] J. M. M. Hall, D. B. Leinweber, B. J. Owen, and R. D. Young, Finite-volume corrections to charge radii, Phys. Lett. B 725, 101 (2013).

[54] J. M. M. Hall, D. B. Leinweber, and R. D. Young, Chiral extrapolations for nucleon electric charge radii, Phys. Rev. D 88, 014504 (2013).

[55] D. B. Leinweber, Lattice QCD evaluation of baryon magnetic-moment sum rules, Phys. Rev. D 45, 252 (1992).
[56] C. Patrignani et al. (Particle Data Group), Review of particle physics, Chin. Phys. C 40, 100001 (2016).

[57] J. M. M. Hall, W. Kamleh, D. B. Leinweber, B. J. Menadue, B. J. Owen, and A. W. Thomas, Light-quark contributions to the magnetic form factor of the $\Lambda(1405)$, Phys. Rev. D 95, 054510 (2017).

[58] C. Lang and V. Verduci, Scattering in the $\pi N$ negative parity channel in lattice QCD, Phys. Rev. D 87, 054502 (2013). 